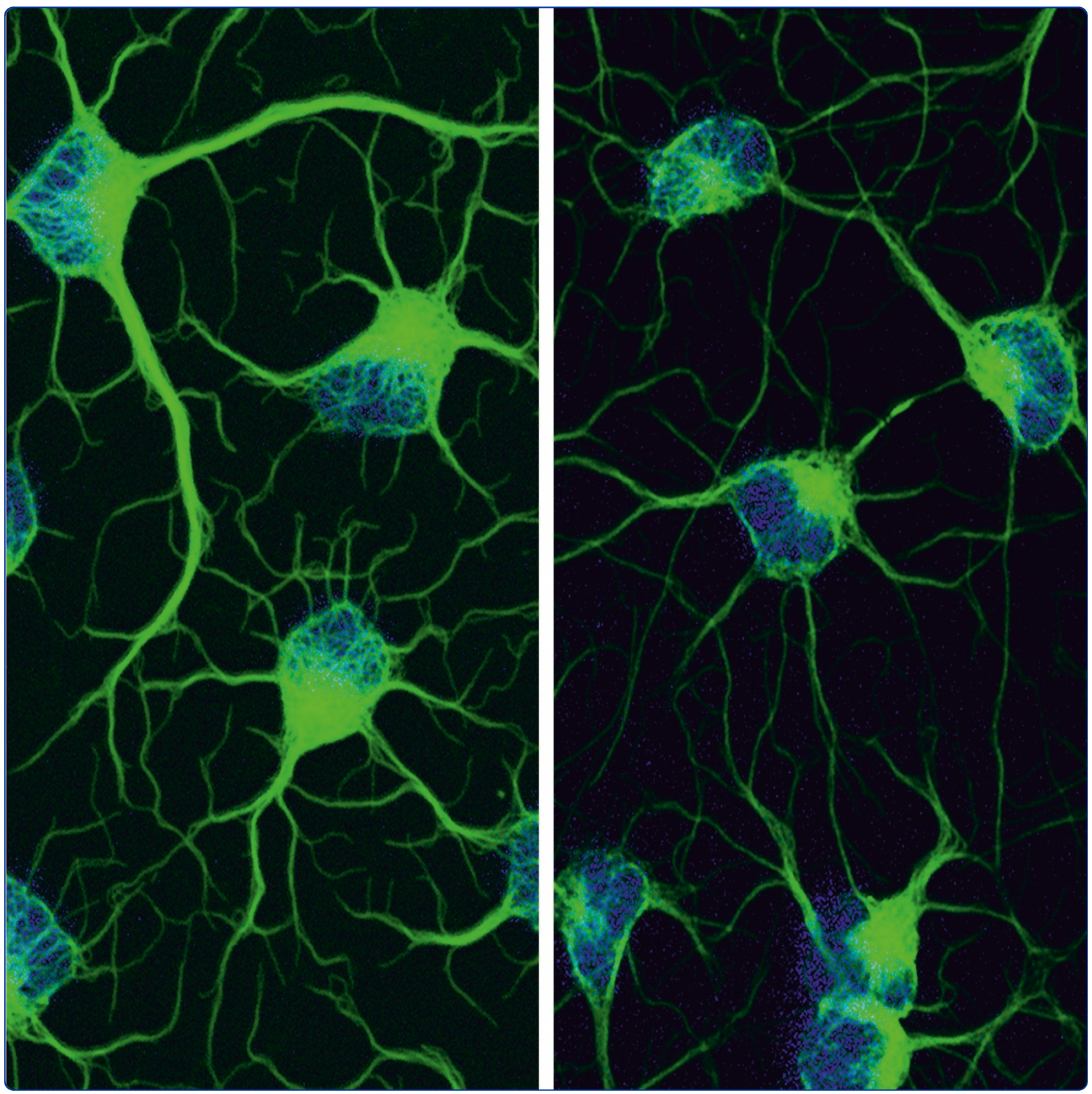

Phosphorylation of collapsin response mediator protein-2 disrupts neuronal maturation in a model of adult neurogenesis: Implications for neurodegenerative disorders crews et al. 


\title{
Phosphorylation of collapsin response mediator protein-2 disrupts neuronal maturation in a model of adult neurogenesis: Implications for neurodegenerative disorders
}

Leslie Crews ${ }^{1}$, Rebecca Ruf ${ }^{1}$, Christina Patrick', Wilmar Dumaop², Margarita Trejo-Morales ${ }^{1}$, Cristian L Achim³ ${ }^{3}$, Edward Rockenstein ${ }^{1}$ and Eliezer Masliah ${ }^{1,2^{*}}$

\begin{abstract}
Background: Recent studies suggest that the pathogenic process in neurodegenerative disorders may disrupt mature neuronal circuitries and neurogenesis in the adult brain. Abnormal activation of CDK5 is associated with neurodegenerative disorders, and recently a critical role for CDK5 in adult neurogenesis has been identified. We have developed an in vitro model of abnormal CDK5 activation during adult hippocampal neurogenesis, and here we used this model to investigate aberrantly phosphorylated downstream targets of CDK5.

Results: Abnormal CDK5 activation in an in vitro model of adult neurogenesis results in hyperphosphorylation of collapsin-response mediator protein-2 (CRMP2) and impaired neurite outgrowth. Inhibition of CDK5, or expression of a non-phosphorylatable (S522A) CRMP2 construct reduced CRMP2 hyperphosphorylation, and reversed neurite outgrowth deficits. CRMP2 plays a role in microtubule dynamics; therefore we examined the integrity of microtubules in this model using biochemical and electron microscopy techniques. We found that microtubule organization was disrupted under conditions of CDK5 activation. Finally, to study the relevance of these findings to neurogenesis in neurodegenerative conditions associated with HIV infection, we performed immunochemical analyses of the brains of patients with HIV and transgenic mice expressing HIV-gp120 protein. CDK5-mediated CRMP2 phosphorylation was significantly increased in the hippocampus of patients with HIV encephalitis and in gp120 transgenic mice, and this effect was rescued by genetic down-modulation of CDK5 in the mouse model.

Conclusions: These results reveal a functional mechanism involving microtubule destabilization through which abnormal CDK5 activation and CRMP2 hyperphosphorylation might contribute to defective neurogenesis in neurodegenerative disorders such as HIV encephalitis.
\end{abstract}

Keywords: neurogenesis, HIV, encephalitis, CRMP2, dpysI2, CDK5, microtubules, neurite outgrowth

\section{Background}

During aging and in the progression of neurodegenerative conditions such as Alzheimer's disease (AD) and HIV-associated neurocognitive disorders, synaptic plasticity and neuronal integrity are disturbed [1-3]. Although the precise mechanisms leading to neurodegeneration in these conditions remain unclear, some common

\footnotetext{
* Correspondence: emasliah@ucsd.edu

'Department of Neurosciences; University of California, San Diego; 9500

Gilman Drive, La Jolla, CA 92093-0624, USA

Full list of author information is available at the end of the article
}

signaling factors have been identified that contribute to the pathogenesis of multiple neurodegenerative processes. One important signaling molecule that may represent a common denominator in several neurodegenerative disorders is cyclin-dependent kinase-5 (CDK5). Previous studies have revealed that dysregulation of CDK5 and its activators p 35 and p 25 contribute to the abnormal accumulation of hyperphosphorylated CDK5 substrates and eventual mature neuronal cell death in AD, HIV-associated neuroinflammatory conditions such as HIV encephalitis (HIVE), and prion-related 
disorders such as scrapie [4-6]. Furthermore, previous studies have shown that levels of CDK5 are increased in the brains of AD [7] and HIVE [8] patients, and in scrapie-infected hamsters [6].

In addition to the alterations in synaptic plasticity in mature neurons in these disorders, recent studies have uncovered evidence suggesting that the pathogenic process in humans and animal models of AD and HIV in the brain might include dysregulation of adult neurogenesis [9-14]. This suggests that neurodegeneration may be characterized by not only a loss of mature neurons but also by a decrease in the generation of new neurons in the neurogenic niches of the adult brain. These cell populations that could be targeted include neural progenitor cells (NPCs) in the subventricular zone (SVZ) and in the dentate gyrus (DG) of the hippocampus. Mechanisms of neurogenesis in the fetal brain have been extensively studied, however less is known about the signaling pathways regulating neurogenesis in the adult nervous system and their role in neurodegenerative disorders.

It is clear that the abnormal activation of CDK5 via calpain-mediated cleavage of p35 into the more stable p25 fragment contributes to the pathogenesis of neurodegenerative conditions such as AD and HIVE [4-6,8], however, previous studies have also demonstrated that physiological CDK5 activity is essential for adult neurogenesis $[15,16]$. Thus, it is possible that abnormal activation of CDK5 and aberrant phosphorylation of its physiological substrates might have detrimental effects on cells residing in the neurogenic niches of the adult brain, and deficits in neurogenesis associated with neurodegeneration might be related to alterations in CDK5 in NPCs. In support of this possibility, we have previously shown that abnormal CDK5 activation impairs neurite outgrowth and neuronal maturation in an in vitro model of adult neurogenesis, and in a mouse model of AD-like neurodegeneration and impaired neurogenesis [17]. However, the downstream regulators mediating CDK5-associated defective neurogenesis are unknown.

In this context, CDK5 may mediate alterations in neurogenesis in $\mathrm{AD}$ and HIVE via aberrant phosphorylation of its substrates, which include cytoskeletal (neurofilaments, nestin) [18] and synaptic proteins (e.g. synapsin) [19], among others. It is possible that CDK5 substrates implicated in toxicity to mature neuronal populations, such as tau, might be involved, however another possibility is that alternative downstream substrates of CDK5 might modulate neurogenesis and neuronal maturation in the adult hippocampus. Elucidating the signaling pathways and downstream molecular targets involved in the dysregulation of neurogenesis is important to fully understand the mechanisms of neuroplasticity in neurodegenerative disorders.

A subset of physiological substrates of CDK5 includes proteins that have been implicated in neurogenesis (doublecortin, nestin) or neuronal development (collapsin-response mediator protein-2 [CRMP2]). Interestingly, in previous reports, CDK5-mediated phosphorylation of CRMP2 has been linked to neurodegeneration in $\mathrm{AD}$ [20-22], and the known role of this protein in growth cone collapse and neuronal development makes it an intriguing candidate for regulating neurogenesis in neurodegenerative disorders. Notably, several recent proteomics-based studies in the brains of human HIV patients have detected differential expression of CRMP2 in human cases with HIVE or HIV-associated dementia $[23,24]$. Since the role of CRMP2 phosphorylation has not been previously examined in the adult neurogenic niche, or in HIV-associated neurodegenerative disorders (HAND), we sought to better understand the potential function of this protein in the molecular mechanisms involved in CDK5-mediated dysregulation of adult neurogenesis using an in vitro model of CDK5 activation in adult hippocampal NPCs [17], and in vivo in the brains of patients with HIV and in an animal model of HIV protein neurotoxicity. Here we demonstrate that aberrant phosphorylation of collapsinresponse mediator protein-2 (CRMP2) is a critical downstream event contributing to impaired neurite outgrowth in an in vitro model of abnormal activation of CDK5 in adult neurogenesis. Using biochemical techniques with genetic and pharmacological manipulation of the $\mathrm{p} 35 / \mathrm{CDK} 5 / \mathrm{CRMP} 2$ signaling pathway, we examined the effects of abnormal activation of CDK5 on neurite outgrowth and CRMP2 phosphorylation in NPC-derived neural progeny. We further investigated the contribution of CDK5-mediated hyperphosphorylation of CRMP2 in neurodegenerative processes in vivo by evaluating expression and phosphorylation of CRMP2 in the neurogenic niche of human brains with HIVE and in gp120 transgenic $(\mathrm{tg})$ mice. These results reveal a role for CRMP2 in impaired neuronal maturation mediated by abnormal CDK5 activation, and identify a potential downstream functional mechanism involving microtubule destabilization that could contribute to impaired neuronal maturation during adult neurogenesis in neurodegenerative conditions.

\section{Results}

CRMP2 phosphorylation is increased in an in vitro model of adult neurogenesis with abnormal CDK5 activation

In order to investigate the molecular mechanisms that might contribute to defective adult neurogenesis in neurodegenerative disorders, we utilized a model system of 
adult neurogenesis, in which activation of CDK5 is induced by virus-mediated overexpression of the CDK5 activator, p35. We have previously shown that these conditions result in dramatically reduced neurite outgrowth of $\beta$-Tubulin-positive processes compared to vehicle-treated controls [17]. At high magnification, cells expressing high levels of p35 had significantly shorter processes and diffuse cytoplasmic $\beta$-Tubulin immunoreactivity compared to vehicle-treated controls (Figure $1 \mathrm{~A}-\mathrm{C})$. This suggests that CDK5 plays a critical role during neuronal maturation of adult hippocampal NPCs, however the downstream mediators of this effect are unknown. It is possible that CDK5 substrates implicated in toxicity to mature neuronal populations, such as tau, might be involved, however it is also possible that alternative targets of CDK5 might modulate neuronal maturation in the adult hippocampus.

CRMP2 has been previously identified as a downstream target of CDK5 and is phosphorylated by this kinase at the Ser522 epitope [25,26]. Although abnormal CDK5 activation has been implicated in neurodegeneration, and CDK5 is critical in the process of adult neurogenesis, the role of CRMP2 in neurogenesis under conditions of abnormal CDK5 activation is unclear. In

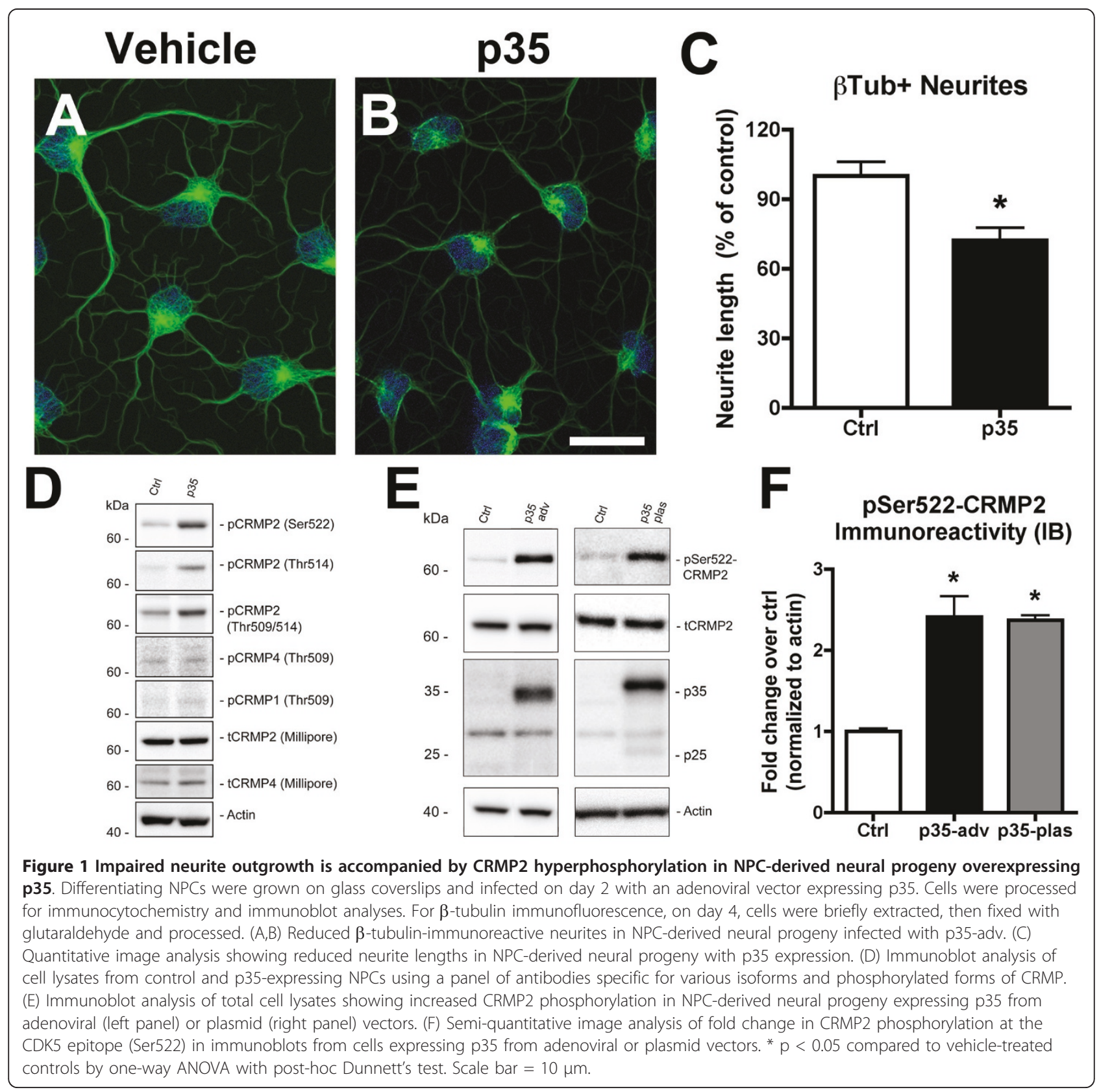


order to investigate whether CRMP2 might be a target of abnormal CDK5 activity in NPC-derived neural progeny over-expressing p35, we performed western blot screening with a panel of antibodies specific for different phosphorylated forms of CRMP2 and related proteins (CRMP4, CRMP1) or total non-phosphorylated forms (Table 1). This analysis confirmed that CRMP2 was hyperphosphorylated at the CDK5-specific epitope (Ser522) in total cell lysates from NPC-derived neural progeny overexpressing p35 compared to control cultures (Figure 1D-F). Increased phosphorylation was also detected at other residues in the $\mathrm{C}$-terminal tail of CRMP2 (Thr509/Thr514) (Figure 1D), however since previous reports have shown that phosphorylation by CDK5 at the Ser522 residue is required as a priming step before GSK3 $\beta$-mediated phosphorylation at the nearby Thr residues can occur $[25,27,28]$, we focused our investigations on the CDK5-specific epitope at Ser522. Total levels of CRMP2 were unchanged in cells overexpressing p35 compared to control cells, and other related proteins from the CRMP family were not differentially phosphorylated under these conditions of CDK5 activation (Figure 1D).

For confirmation that the observed increase in CRMP2 phosphorylation was not due to virus-specific effects, we performed similar experiments using an alternative vector for $\mathrm{p} 35$ overexpression. For this purpose, we performed immunoblot analysis in parallel with NPC-derived neural cells infected with p35-adv or cells transfected with a plasmid expressing myc-tagged p35. We found that phosphorylation of the CDK5 epitope Ser522-CRMP2 was significantly upregulated in cells overexpressing p35 via both adenoviral vector or plasmid transfection compared to uninfected controls (Figure 1E,F). For all subsequent experiments, results shown are from experiments where untagged p35 was overexpressed using the adenoviral vector.
To further verify that CRMP2 is a target of p35mediated abnormal activation of CDK5, we used siRNA to knock down expression of the endogenous CRMP2 protein (siCRMP2), and performed immunoblot analysis to assess levels of pSer522-CRMP2 and total CRMP2 (Additional File 1, Figure S1). We found that transfection with siCRMP2 decreased total levels of CRMP2 expression by approximately $80 \%$ compared to controls in both untreated and p35-expressing NPC-derived neural progeny (Additional File 1, Figure S1 A,B). Moreover, transfection with siCRMP2 dramatically reduced levels of pSer522-CRMP2 by over $90 \%$ in control cells, and completely reversed the hyperphosphorylation of CRMP2 induced by p35 expression in NPC-derived neural progeny (Additional File 1, Figure S1 A,C). Taken together, these studies confirmed that CRMP2 was hyperphosphorylated at the CDK5-specific residue (Ser522) under conditions of abnormal CDK5 activation.

\section{CRMP2 phosphorylation can be reversed in vitro with pharmacological or genetic down-regulation of CDK5}

Our previous observations showed that abnormal CDK5 activation in NPC-derived neural progeny impaired neurite outgrowth and cell maturation [17] (Figure 1). Moreover, down-regulating CDK5 activity or expression reversed the neurite outgrowth deficits [17]. In order to determine whether CDK5-mediated CRMP2 phosphorylation might contribute to these effects, we used a pharmacological inhibitor of CDK5 (Roscovitine) or an siRNA-based approach to down-regulate CDK5 activity or expression, respectively, and assessed levels of CRMP2 phosphorylation by immunocytochemistry and immunoblot. For this purpose, differentiating NPCs were pre-treated with Roscovitine, or transfected with siRNA targeting CDK5 for 6 hrs, followed by infection with adenovirus expressing p35. We have previously qualified and tested the siRNA targeting CDK5 for

Table 1 Antibodies tested and reactive epitopes against phosphorylated and total CRMP proteins.

\begin{tabular}{lllll}
\hline Antibody & Epitope & Company & Species & Reactivity \\
\hline CRMP2 & pThr514 & Cell Signaling & Rabbit & Human, mouse, rat \\
\hline CRMP2 & pThr514 & Kinasource & Sheep & Human, mouse \\
\hline CRMP2 & pThr509, pThr514 & Kinasource & Sheep & Human, mouse, rat \\
\hline CRMP2 & pSer522 & ECM Biosciences & Rabbit & Human, mouse, rat \\
\hline CRMP2 & pSer522 & Kinasource & Sheep & Human, mouse, rat \\
\hline CRMP2 & pThr555 & ECM Biosciences & Rabbit & Human, mouse, rat \\
\hline CRMP2 & total (C-terminus) & ECM Biosciences & Rabbit & Human, mouse, rat \\
\hline CRMP2 & total (peptide) & Millipore & Rabbit & Rat \\
\hline CRMP2 & total (recombinant) & Kinasource & Sheep & Human, mouse CRMP1,2,4 \\
\hline CRMP1 & pThr509 & Kinasource & Sheep & Human \\
\hline CRMP4 & pThr509 & Kinasource & Sheep & Human, mouse, rat \\
\hline CRMP4 (TUC4) & total (peptide) & Millipore & Rabbit & Human, mouse, rat
\end{tabular}


efficacy in our system using two unique siRNA sequences [17].

Notably, under conditions of reduced CDK5 activity or expression in $\mathrm{p} 35$-expressing NPC-derived neural progeny, reduced levels of CRMP2 phosphorylation were detected, as demonstrated by immunocytochemical (Figure 2A-G) and immunoblot analyses (Figure 2H,I) with an antibody against pSer522-CRMP2. Roscovitine treatment in the absence of p35 over-expression does not appear to have a significant effect on levels of CRMP2 phosphorylation (Figure 2). It is possible that this could be due to alternative kinases phosphorylating CRMP2, however as CDK5 is the only kinase known at this time to phosphorylate CRMP2 at Ser522, another mechanism

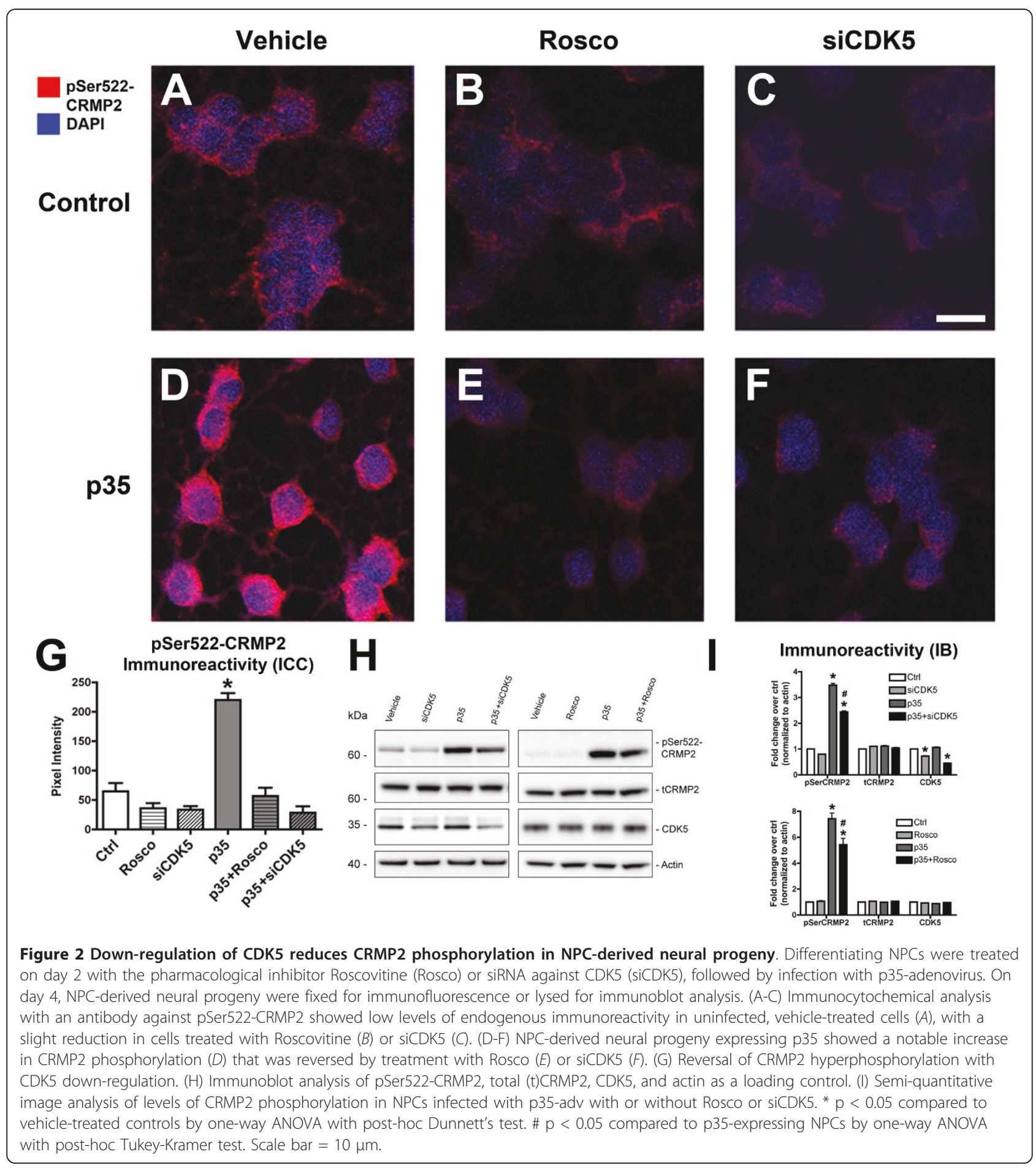


must be at play. Previous studies have shown that Ser522-phosphorylated CRMP2 is highly resistant to desphosphorylation by protein phosphatases [29]. Therefore, it is likely that the CRMP2 that is already phosphorylated by basal CDK5 activity prior to roscovitine treatment remains phosphorylated and stable, while roscovitine treatment of cells with exogenous p35 expression more effectively modulates levels of CRMP2 phosphorylation at the Ser522 residue. Taken together, these results support the possibility that abnormal CDK5 activation targets CRMP2 for hyperphosphorylation, and this can be reversed by down-modulating abnormal CDK5 activity.

\section{Expression of a non-phosphorylatable CRMP2 construct reduces CRMP2 phosphorylation and rescues neurite outgrowth defects in adult NPCs with abnormal CDK5 activation}

In order to determine whether CRMP2 phosphorylation is directly related to the neurite outgrowth defects observed in our cellular model of abnormal CDK5 activation in adult neurogenesis, we utilized site-directed mutagenesis to generate a construct encoding a mutant (S522A) form of CRMP2 (S522A-CRMP2) that is not phosphorylatable by CDK5 at the Ser522 epitope (Additional File 1, Figure S2 A). Immunoblot analysis confirmed that transfection of differentiating NPCs with the S522A-CRMP2 construct or WT-CRMP2 resulted in similar levels of total CRMP2 expression (Additional File 1, Figure S2 B). In contrast, levels of pSer522CRMP2 immunoreactivity were considerably lower in NPC-derived neural progeny expressing S522A-CRMP2 compared to WT-CRMP2-transfected controls (Additional File 1, Figure S2 B,C). Controls including transfection agent (Lipofectamine) alone or CMV-GFP vector control showed that levels of pSerCRMP2 and total CRMP2 immunoreactivity were similar to non-transfected controls (Additional File 1, Figure S2 B,C).

In order to assess whether down-regulating CDK5mediated phosphorylation of CRMP2 at Ser522 by expression of a mutant construct might have a protective effect on the neurite alterations observed in NPCderived neural progeny with abnormal CDK5 activity, neurite outgrowth analysis was performed in cultured cells immunolabeled with an antibody against $\beta$-Tubulin (Figure 3A-F). This demonstrated that expression of the mutant S522A-CRMP2 construct rescued the neurite deficits in NPC-derived neural progeny expressing p35, whereas expression of the WT-CRMP2 construct was not protective under these conditions (Figure 3A-G).

Immunoblot analysis confirmed that expression of S522A-CRMP2 reduced levels of CRMP2 phosphorylation in $\mathrm{p} 35$-expressing NPC-derived neural progeny compared to WT-CRMP2. We detected a slight apparent increase in pSer-CRMP2 immunoreactivity in lysates from cells expressing the non-phosphorylatable mutant S522A-CRMP2 construct. Higher resolution gel electrophoresis studies indicated that while CRMP2 hyperphophorylation induced by p35 overexpression resulted in a gel mobility shift that was consistent with a change in phosphorylation state [27], no such shift was detected with overexpression of the S522A-CRMP2 mutant construct (data not shown). It is possible that the apparent increase in immunoreactivity with S522ACRMP2 over-expression could be due to a mild change in protein folding induced by the mutation, causing a slight affinity of the pSerCRMP2 antibody for the mutant unphosphorylated human protein. Levels of total CRMP2 were similar in cells transfected with either the WT or mutant constructs (Figure 3H,I). Levels of CRMP2 phosphorylation were expressed as ratios of pCRMP2/tCRMP2 immunoreactivity because overexpression of the CRMP2 constructs increases total levels of CRMP2. Since over-expression of WT-CRMP2 is known to promote neurite outgrowth in neuronal cell lines [30], when WT-CRMP2 is overexpressed and phosphorylation is increased, there are increased quantities of both total and phospho-CRMP2. Thus, under conditions of CRMP2 overexpression, the ratio of phospho-CRMP2/total-CRMP2 may be the most accurate representation of relative CRMP2 phosphorylation that impacts neurite outgrowth. Taken together, these data support the possibility that phosphorylation of CRMP2 by CDK 5 at the Ser522 epitope contributes to impaired neurite outgrowth under conditions of abnormal CDK5 activation in cultured NPCs.

\section{Tubulin distribution and microtubule organization are disturbed in NPC-derived neural progeny with abnormal CDK5 activation}

Previous studies have shown that phosphorylation of CRMP2 regulates its interactions with tubulin heterodimers [30]. In light of our observations thus far, it is possible that CDK5-mediated hyperphosphorylation of CRMP2 in our in vitro model might impair neurite outgrowth by disrupting microtubule polymerization in developing neuronal processes. First, to assess the distribution of polymerized tubulin in live cell cultures, Tubulin Tracker reagent was applied to NPC-derived neural progeny after four days of differentiation under control and activated CDK5 conditions (Figure 4A,B). In cultures infected with p35-adv, reduced neurite complexity was apparent compared to uninfected controls. Furthermore, under control conditions, the distribution of polymerized tubulin appeared to be localized to cell processes, and characterized by well-defined bundled tubulin fibers (Figure 4A). In contrast, under conditions of abnormal CDK5 activation, the polymerized tubulin 


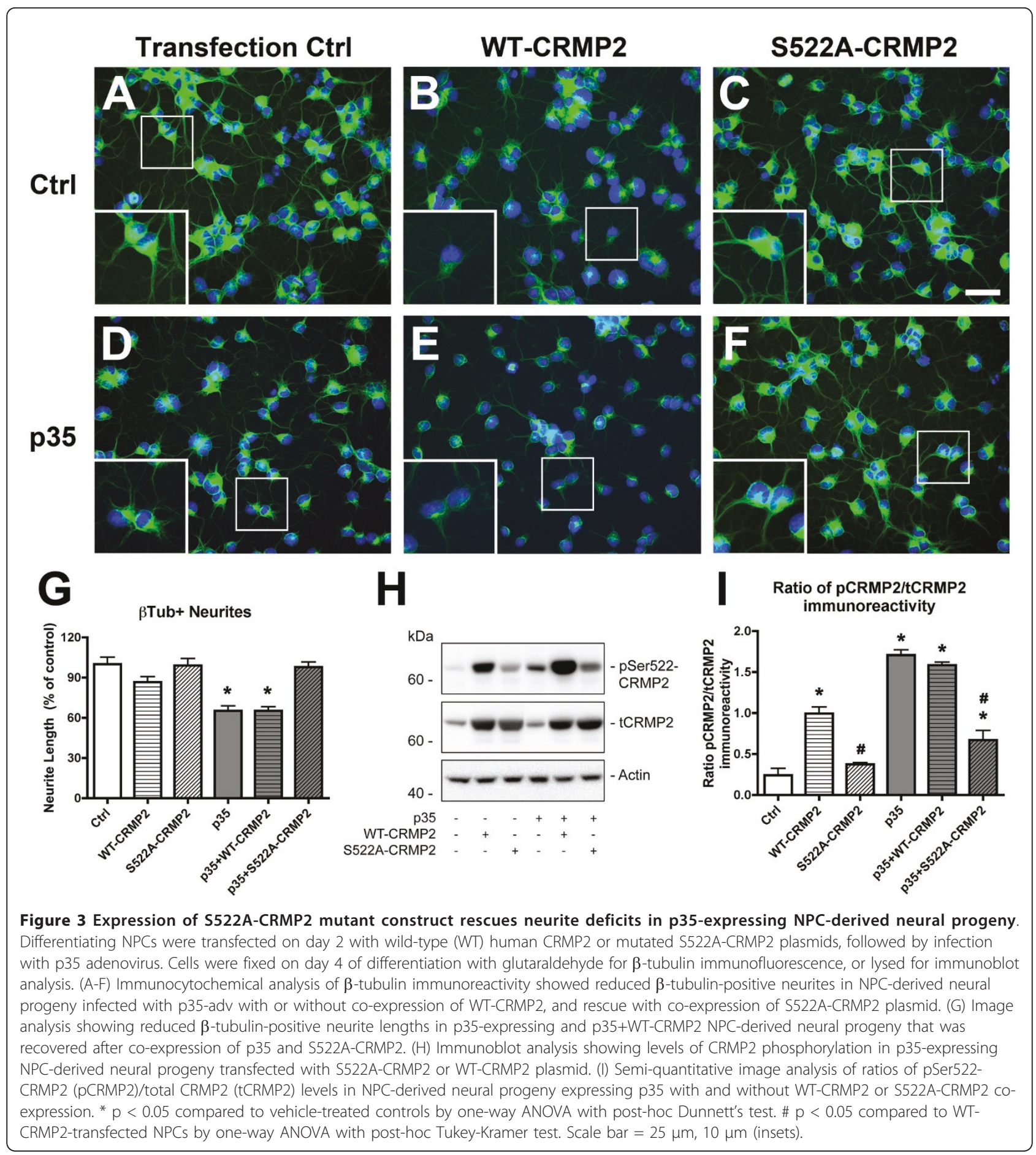

pool appeared to be comprised of more diffuse, fine, mesh-like tubulin fibers localized to the cytoplasm in cells infected with p35-adv (Figure 4B).

To further investigate the underlying ultrastructural basis of the neurite alterations observed in p35-overexpressing NPC-derived neural progeny, cells on coverslips were fixed in glutaraldehyde and analyzed by electron microscopy (Figure 4C-F). These studies showed that control NPC-derived neural progeny displayed long electron-dense microtubule structures distributed along neuritic processes in a parallel, organized arrangement (Figure 4C,D). In contrast, in 


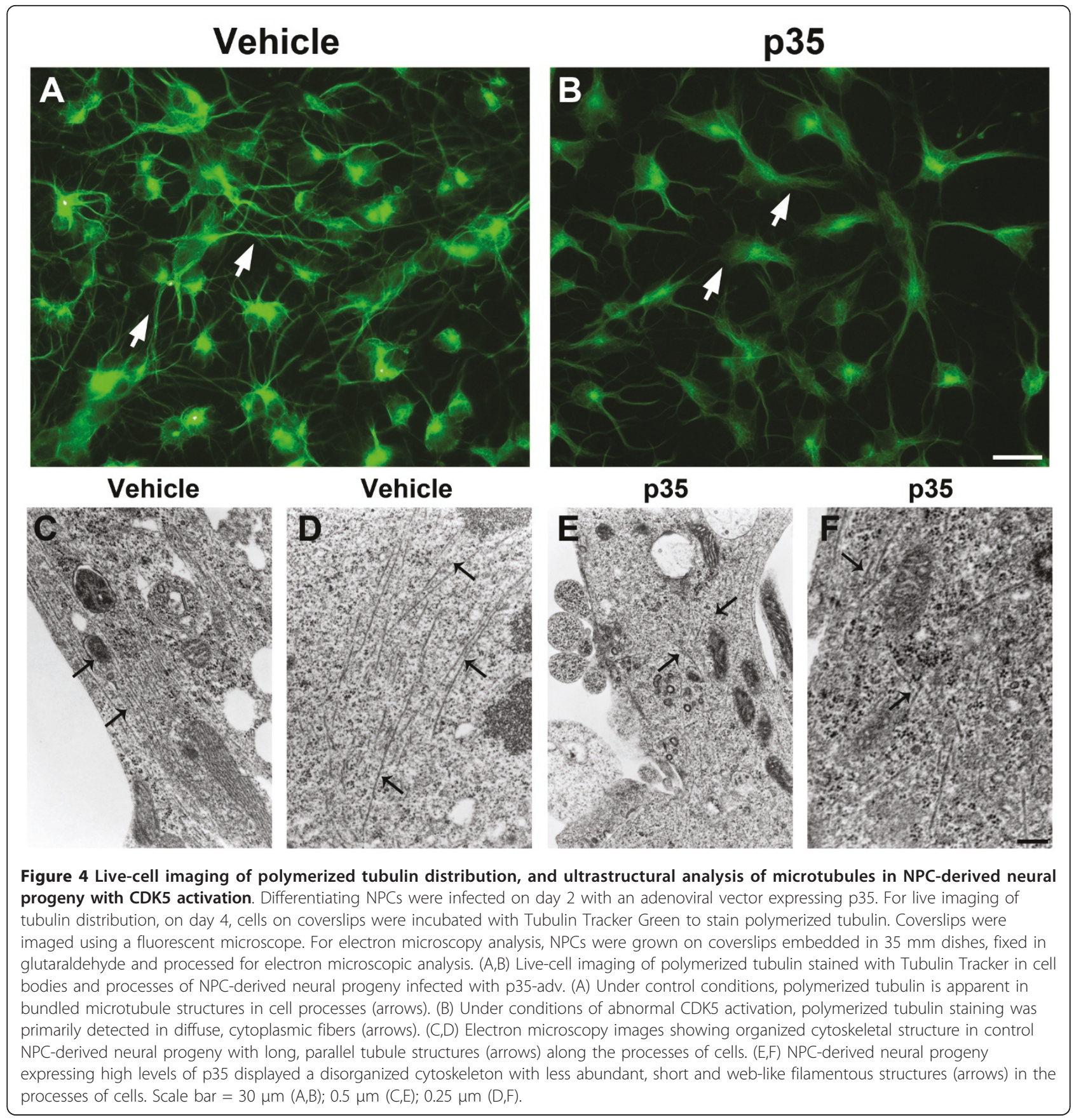

the processes of p35-expressing NPC-derived neural progeny, filamentous and tubule-like structures were less abundant, and those that were observed were shorter and more disorganized than in control cells (Figure 4E,F). These results support the possibility that abnormal activation of CDK5 and hyperphosphorylation of CRMP2 during neurogenesis in neurodegenerative disorders might impair microtubule polymerization and disrupt the organization of these important structural components of the cytoskeleton.
NPC-derived neural progeny with abnormal CDK5 activation show impaired microtubule polymerization following exposure to microtubule destabilizing agents To further investigate the process of microtubule polymerization in NPC-derived neural progeny, we performed a series of nocodazole washout experiments to visualize the formation of microtubules over a period of 10-30 mins. For this purpose, NPC-derived neural progeny were incubated with nocodazole for $3 \mathrm{hrs}$, followed by no washout, or washout for 10-30 mins (Figure 5A- 


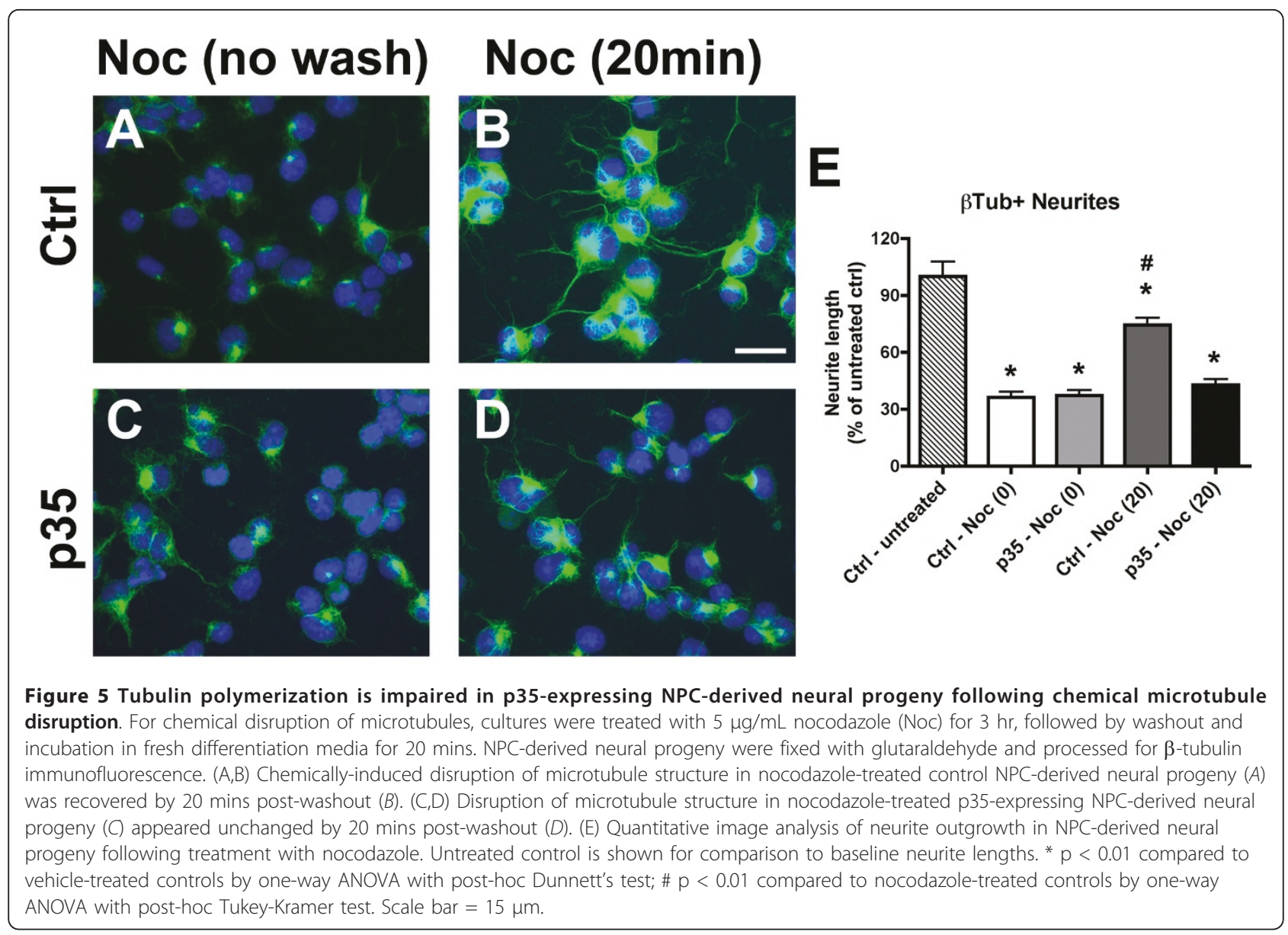

D). Cells were permeabilized during glutaraldehyde fixation to allow visualization of intact microtubules, then processed for $\beta$-tubulin immunofluorescence and imaged on a fluorescent microscope. This showed, as expected, that directly following nocodazole treatment (no washout), both control and p35-expressing NPCderived neural progeny displayed a dramatic reduction of $\beta$-tubulin immunoreactivity, and microtubule structure was almost completely ablated in both groups (Figure $5 \mathrm{~A}, \mathrm{C}$ ). Twenty minutes after nocodazole washout, in both control and NPC-derived neural progeny expressing high levels of p35, we observed the appearance of perinuclear microtubule organizing centers (Figure 5B, D).

By 20 minutes post-washout, control nocodazole-treated cultures (Figure 5B) regained approximately $75 \%$ of the neurite lengths compared to untreated control cultures (Figure 5E), while p35-expressing cells only displayed diffuse $\beta$-tubulin immunoreactivity in processes (Figure 5D), and no significant increase in neurite lengths (Figure 5E). Only very fine processes were detected under these conditions up to 30 minutes postwashout (data not shown). These results are consistent with the possibility that abnormal activation of CDK5 in the pathogenesis of neurodegenerative disorders might disrupt adult neurogenesis by impairing microtubule polymerization and neurite outgrowth.

\section{Increased CRMP2 phosphorylation in a model of HIV-}

protein mediated activation of CDK5 in adult neurogenesis, and reversal with CDK5 siRNA

We and others have shown that CDK5 signaling is dysregulated in patients with HIVE and in animal and in vitro models of HIV-associated neurotoxicity [8,31]. These studies demonstrate that the HIV protein gp 120 contributes to calpain-mediated activation of CDK5 and downstream hyperphosphorylation of CDK5 substrates such as tau in mature neuronal populations [8,31]. In this context, we sought to examine the possibility that HIV proteins could modulate CRMP2 phosphorylation in a similar manner to what we observed in our in vitro model of p35-directed abnormal CDK5 activation in adult neurogenesis. For this purpose, NPC-derived neural progeny were exposed to recombinant HIVgp120 protein for 24 hrs on day 3 of differentiation. Double-immunolabeling analysis with antibodies against 
the immature neuronal marker $\beta$-III Tubulin and pSer522-CRMP2 revealed that gp120 treatment resulted in upregulated CRMP2 phosphorylation, and this effect was accompanied by reduced $\beta$-III Tubulin immunoreactivity (Figure 6A-F,M,N). siRNA experiments to knock down CDK5 expression showed that these effects could be rescued by down-modulating CDK 5 expression (Figure 6G-N). Similar to what we observed previously using the CDK5-targeting siRNA (Figure 2 and [17]), in NPCs expressing CDK5 siRNA alone or in combination with gp120 treatment, CDK5 levels were reduced by approximately 50-60\% (data not shown). Taken together, these studies in an in vitro model of adult neurogenesis reveal that abnormal activation of CDK 5 mediated by either p35 overexpression or HIV-gp120 treatment triggers hyperphosphorylation of CRMP2, which may contribute to microtubule destabilization and impaired neurogenesis and neuronal maturation in the mature brain.

\section{CRMP2 phosphorylation is increased and dendritic} maturation is impaired in primary neuronal cultures with abnormal CDK5 activation

Since our results in NPC-derived neural progeny suggest that abnormal CDK5 activation and hyperphosphorylation of CRMP2 contributes to impaired neurite outgrowth during neurogenesis, we sought to determine whether these factors might also play a role in neurite maturation in mature neuronal cells. For this purpose, we infected primary rat hippocampal neurons with p35-

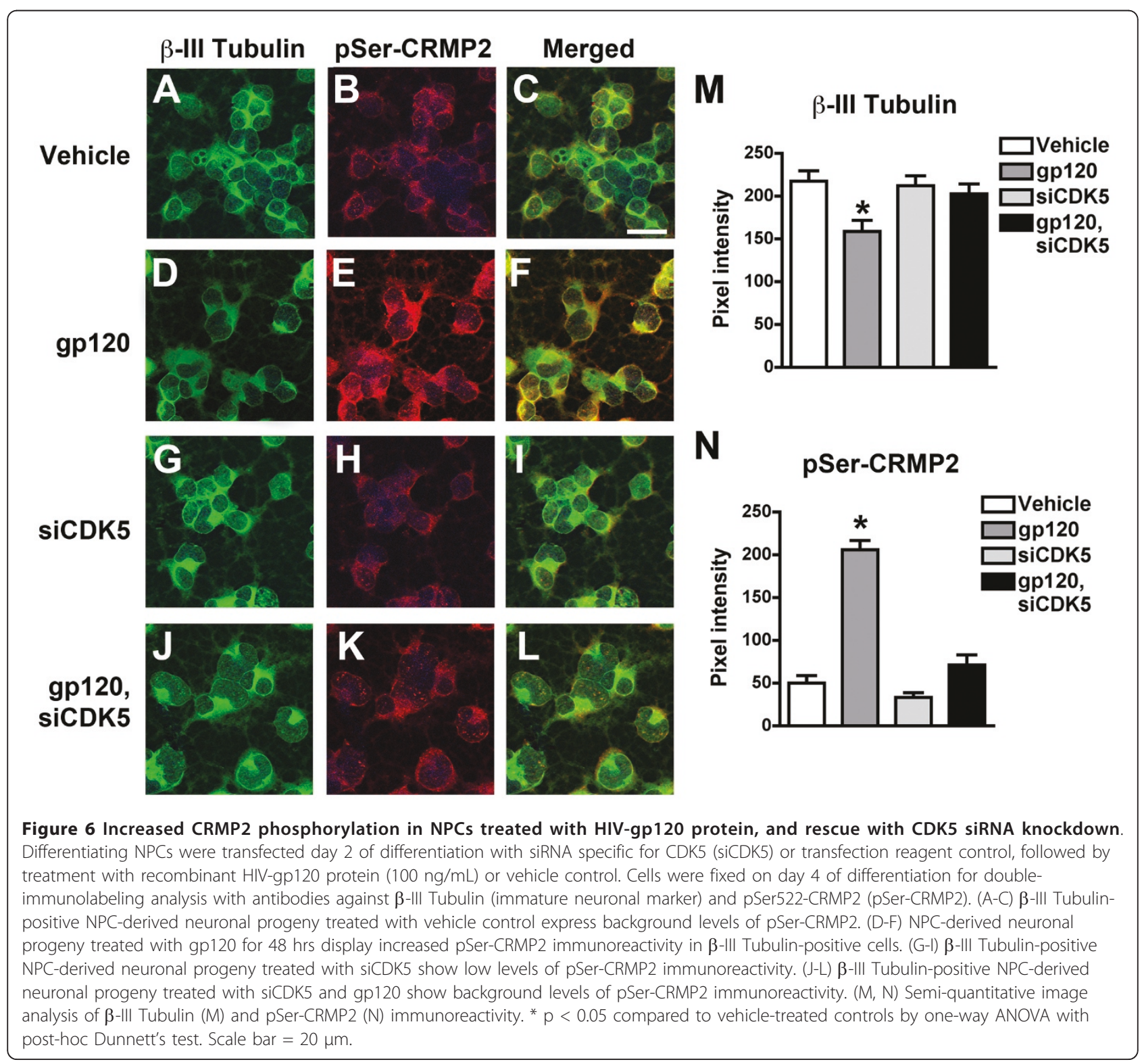


adv, and two days later, levels of CRMP2 phosphorylation and dendritic integrity were analyzed by immunocytochemistry and immunoblot. Similar to NPC-derived neural progeny, primary hippocampal neurons overexpressing p35 displayed increased pSer522-CRMP2 immunoreactivity compared to uninfected controls (Figure 7A-F, M-P). Interestingly, under control conditions, primary neurons expressed higher levels of pSer522CRMP2 than NPCs, and thus the change in pSer522CRMP2 immunoreactivity with p35 overexpression was less dramatic in primary neuronal cultures than in NPCs (40-50\% increase in CRMP2 phosphorylation in primary neurons compared to 3-4 fold in NPC-derived neural progeny). However, similar to what was observed in NPCs, in primary neurons increased p35 expression and CRMP2 phosphorylation was accompanied by reduced neurite maturation and dendritic complexity, as shown by immunolabeling with an antibody against microtubule-associated protein-2 (MAP2, dendritic marker) (Figure 7A-F, M, N). Similar effects were observed in primary neuronal cultures exposed to HIV-gp120 protein for $24 \mathrm{hrs}$ alone or in combination with p35 overexpression (Figure 7G-P). Under these conditions, extensive damage to MAP2-immunoreactive processes was visible, and pSer522-CRMP2 immunoreactive deposits were detected in varicosities along the neuronal processes (Figure 7G-L). Taken with the observations from experiments with NPC-derived neural progeny, these results suggest that abnormal CDK5 activation and subsequent CRMP2 hyperphosphorylation may have deleterious effects on neurite outgrowth in both immature and mature neuronal populations in the adult brain. Thus, the role of CRMP2 hyperphosphorylation in neurodegenerative disorders where abnormal CDK5 activation contributes to the pathogenesis of the disease warrants investigation.

\section{CRMP2 is hyperphosphorylated in the brains of patients with HIVE}

To further evaluate the potential contribution of CRMP2 to neurogenic alterations in the pathogenesis of HIV in the brain, we examined expression levels and patterns of immunoreactivity of CRMP2 in the hippocampus of HIV patients with and without encephalitis (patient demographics and clinic-pathological characteristics presented in Table 2). In support of a role for CRMP2 in the neurogenic niche of the adult hippocampus, double-labeling immunocytochemical analysis with antibodies against doublecortin (DCX, a marker of immature neuroblasts) and CRMP2 revealed that CRMP2 is expressed throughout the hippocampal DG in the brains of patients with HIVE (Figure 8A-C). A subset of cells in the subgranular zone (SGZ) coexpressed both DCX and CRMP2, suggesting that endogenous neuronal progenitor cells in the adult hippocampus could be sensitive to CDK5-mediated hyperphosphorylation of CRMP2 (Figure 8A-C).

To determine whether CRMP2 phosphorylation or expression is altered in the brains of patients with HIVE, immunocytochemical analysis was performed with antibodies against total CRMP2 and pSer522CRMP2 in hippocampal sections from HIV+ (no encephalitis) and HIVE brains (Figure 9). Both total CRMP2 and pSer522-CRMP2 displayed a granular expression pattern in the granular cell layer and subgranular zone of the hippocampal dentate gyrus. Consistent with our previous report that CDK5 expression and activity is increased in the brains of patients with HIVE [8], image analysis revealed that overall CRMP2 and phosphorylated CRMP2 (Ser522 epitope) levels were significantly increased in the hippocampus of HIVE brains compared to HIV+ controls without encephalitis (Figure 9A-J). Numerous intensely CRMP2-immunoreactive nuclear profiles were also detected in the SGZ of HIVE brains (Figure 9D,I), suggesting that in the pathogenesis of HIVE, cells in this neurogenic region are sensitive to CDK5-mediated upregulation of CRMP2 phosphorylation.

To further examine overall expression levels of CRMP2 in the brains of HIVE patients, immunoblot analysis was performed with brain homogenates from HIV+ (no encephalitis) controls and HIVE patients (Additional File 1, Figure S3). This analysis revealed that levels of phosphorylated (Ser522) CRMP2 were significantly upregulated in HIVE patients compared to nonencephalitic HIV+ controls (Additional File 1, Figure S3 A,B). In fact, while some non-encephalitis cases displayed only very low levels of immunoreactivity with the pSer522-CRMP2 antibody, every one of the HIVE cases examined showed robust pSer522-CRMP2 immunoreactivity by immunoblot (Additional File 1, Figure S3 A).

\section{Hyperphosphorylated CRMP2 is detected in the brains of gp120 tg mice, and is reversed with genetic down- modulation of CDK5 expression}

Similar to what we observed in the hippocampus of HIVE patients, CRMP2 and pSer522-CRMP2 immunoreactivity were detected throughout the DG in immunolabeled sections from the brains of non-transgenic (nontg) and gp120 tg mice (Figure 10). In support of a role for CRMP2 in this neurogenic region, double-labeling immunocytochemical analysis with antibodies against DCX and CRMP2 revealed that CRMP2 is expressed throughout the hippocampal DG in the brains of gp120 tg mice (Figure 8D-F). Levels of total CRMP2 and pSer522-CRMP2 immunoreactivity were increased in the hippocampus of gp120 tg mice compared to nontg controls (Figure 10A,B,E,F,G,J), indicating that 


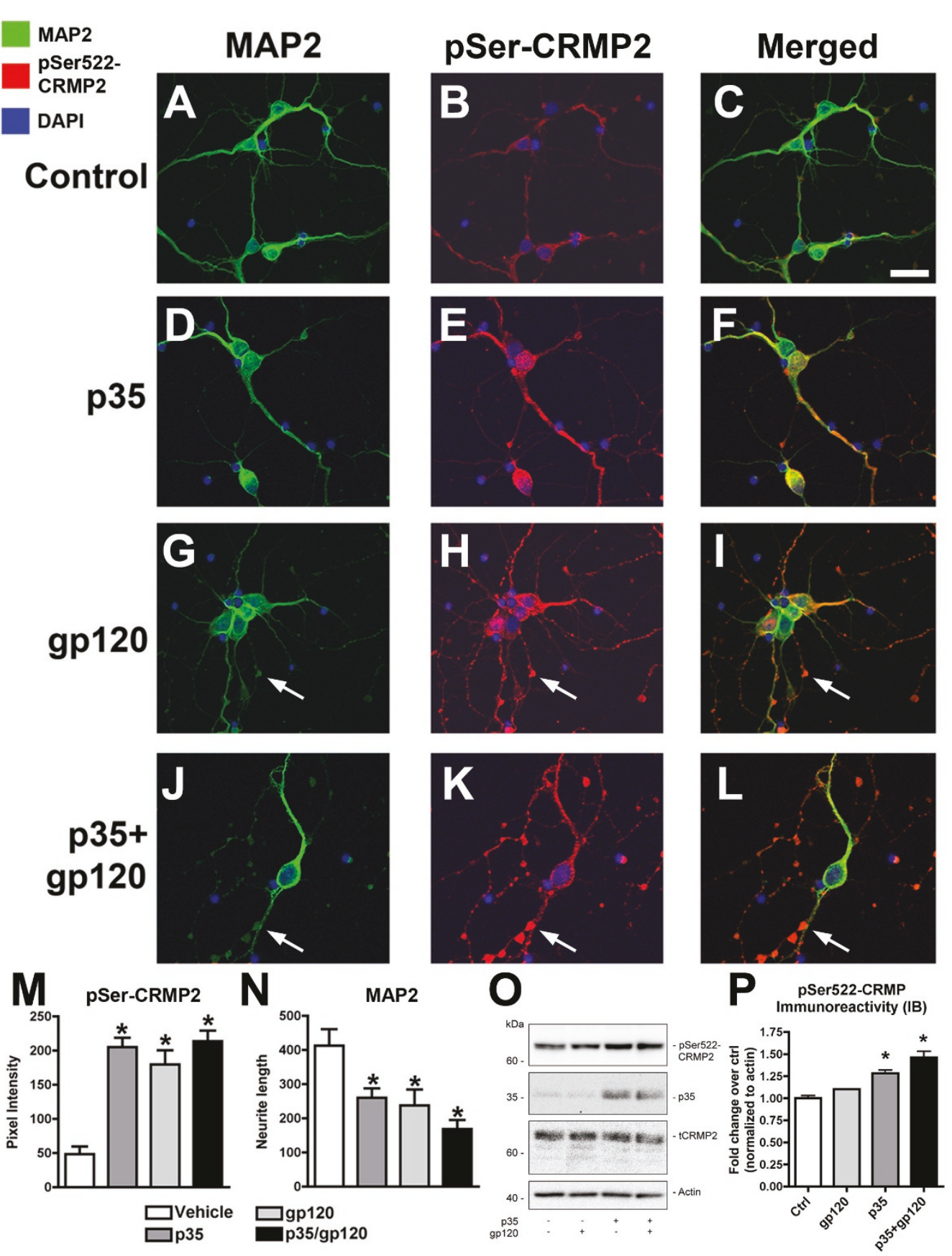

Figure 7 CRMP2 is hyperphosphorylated and dendritic development is impaired in primary neurons over-expressing p35 alone or in combination with HIV-gp120 protein treatment. Primary rat hippocampal neurons were infected two days after plating with p35-adv, followed by treatment with recombinant HIV-gp120 protein or vehicle control. Cells were fixed on day 4 of differentiation for doubleimmunolabeling analysis with antibodies against MAP2 (dendritic marker) and pSer522-CRMP2 (pSer-CRMP2). (A-C) MAP2-positive primary neurons treated with vehicle control express background levels of pSer-CRMP2. (D-F) Primary neurons infected with p35-adv display increased pSer-CRMP2 immunoreactivity and reduced MAP2-immunoreactive dendrites. (G-I) Primary neurons treated with recombinant HIV-gp120 display increased pSer-CRMP2 immunoreactivity and reduced MAP2-immunoreactive dendrites, with an accumulation of pSer-CRMP2 immunoreactivity in varicosities along the neuronal processes (arrows). (J-L) Primary neurons infected with p35-adv and treated with recombinant HIV-gp120 display increased pSer-CRMP2 immunoreactivity and extensive damage to MAP2-immunoreactive dendrites, with an abundant accumulation of pSer-CRMP2 immunoreactivity in varicosities along the neuronal processes (arrows). (M, N) Semi-quantitative image analysis of pSer-CRMP2 (M) and MAP2 (N) immunoreactivity. (O) Immunoblot analysis of total cell lysates showing increased CRMP2 phosphorylation in primary hippocampal neurons infected with p35-adv or treated with HIV-gp120 protein. (P) Semi-quantitative image analysis of fold change in CRMP2 phosphorylation at the CDK5 epitope (Ser522) in immunoblots from cells expressing p35-adv or treated with gp120. ${ }^{*} p<0.05$ compared to vehicle-treated controls by one-way ANOVA with post-hoc Dunnett's test. Scale bar $=20 \mu \mathrm{m}$. 
Table 2 Demographic and clinico-pathological characteristics of HIV+ cases with and without HIV encephalitis.

\begin{tabular}{cccccc}
\hline Neuropathology diagnosis & Age & Gender & PM time (hrs) & Brain weight & Cause of Death \\
\hline Normal & 55 & M & 25 & 1220 & Heart failure \\
\hline Normal & 53 & M & 18 & 1410 & Bespiratory failure \\
\hline Normal & 47 & M & 12 & 1200 & Hypotensive shock \\
\hline Normal & 35 & M & 9 & 1440 & Respiratory failure \\
\hline Normal & 36 & F & 24 & 1370 & Cryptococcal pneumonia \\
\hline Normal & 49 & M & 30 & 1350 & Cardiac infarction \\
\hline Normal & 54 & $M$ & 14 & 1220 & Complications of HIV and HCV co-infection \\
\hline Normal & 67 & $M$ & 15 & 1440 & Organ failure \\
\hline HIV encephalitis & 34 & $M$ & 30 & 1370 & Respiratory failure \\
\hline HIV encephalitis & 39 & $M$ & 19 & 1510 & Bacterial pneumonia \\
\hline HIV encephalitis & 37 & $M$ & 5 & 1040 & Cachexia \\
\hline HIV encephalitis & 55 & $M$ & 15 & 1230 & AlDS \\
\hline HIV encephalitis & 41 & $M$ & 12 & 1350 & Sepsis \\
\hline HIV encephalitis & 43 & $M$ & 6 & 1410 & Undetermined
\end{tabular}

$\mathrm{PM}=$ post-mortem time, $\mathrm{HCV}=$ Hepatitis $\mathrm{C}$ virus

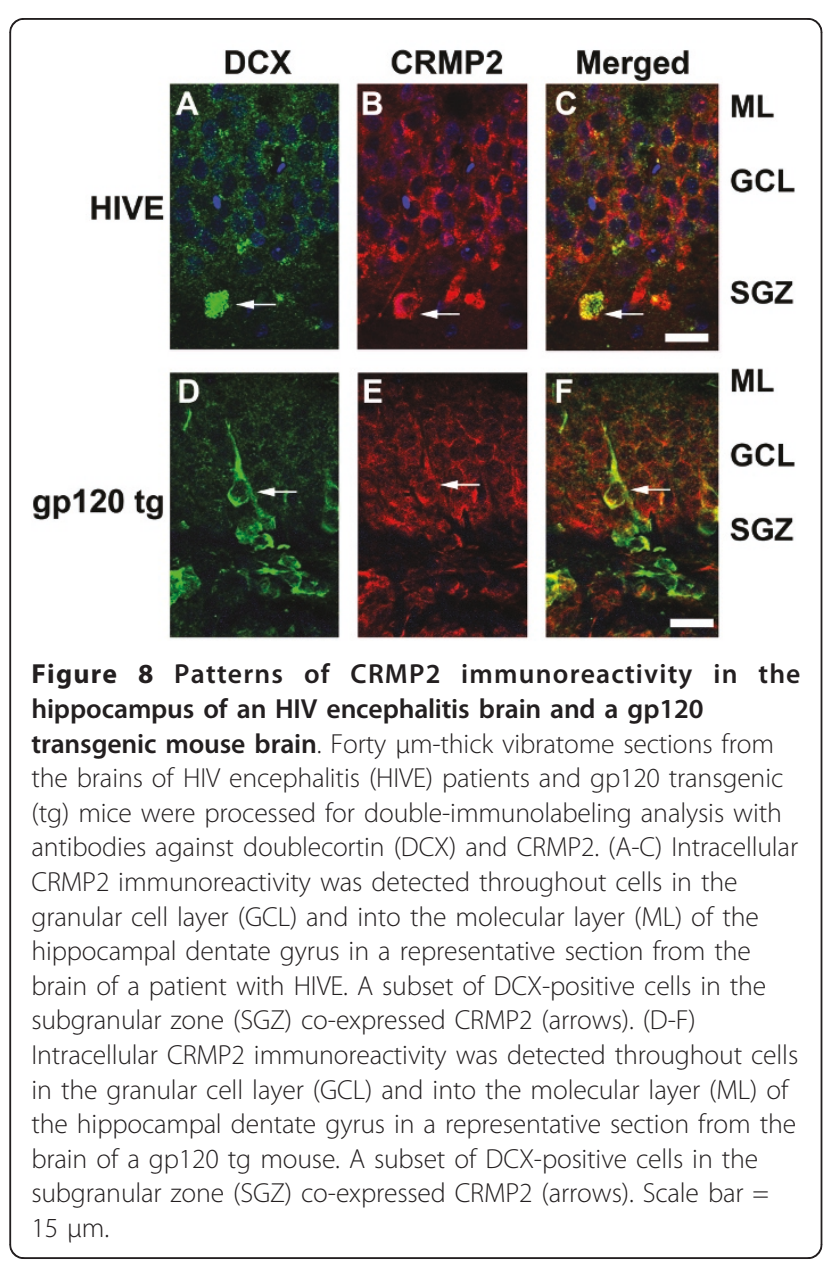

CDK5 activation in this model contributes to abnormal phosphorylation of CRMP2.

In our in vitro model of abnormal CDK5 activation in NPC-derived neural progeny, we found that CDK5directed CRMP2 hyperphosphorylation could be reversed by knocking down CDK5 expression or activity (Figure 2). Therefore, we sought to determine if this effect could also be rescued in vivo in gp120 tg mice crossed with a CDK5 heterozygous-deficient (CDK5+/-) line. Immunocytochemical analysis revealed that this was the case, as gp120 tg/CDK5+/- animals displayed levels of CRMP2 (Figure 10A-E) and pSer522-CRMP2 (Figure 10F-J) immunoreactivity that were comparable to nontg controls.

\section{Discussion}

Abnormal CDK5 activity has been shown to contribute to neurotoxicity during the pathogenesis of several neurodegenerative disorders, including HIV neurotoxicity, $\mathrm{AD}$, and prion-related encephalopathies. In the progression of these disorders, activation of CDK5 may trigger a cascade of hyperphosphorylation of downstream targets, and subsequent modulation of critical cellular functions. Most studies have focused on the role of tau hyperphosphorylation in mediating the neurodegenerative effects of CDK5 and other aberrantly activated kinases in mature neurons $[8,32,33]$. However, considering the myriad substrates phosphorylated by CDK5, other downstream targets may be involved, particularly in distinct functions such as neurogenesis and neuronal maturation. In this context, we report that dysregulation of the growth cone signaling protein CRMP2 by 

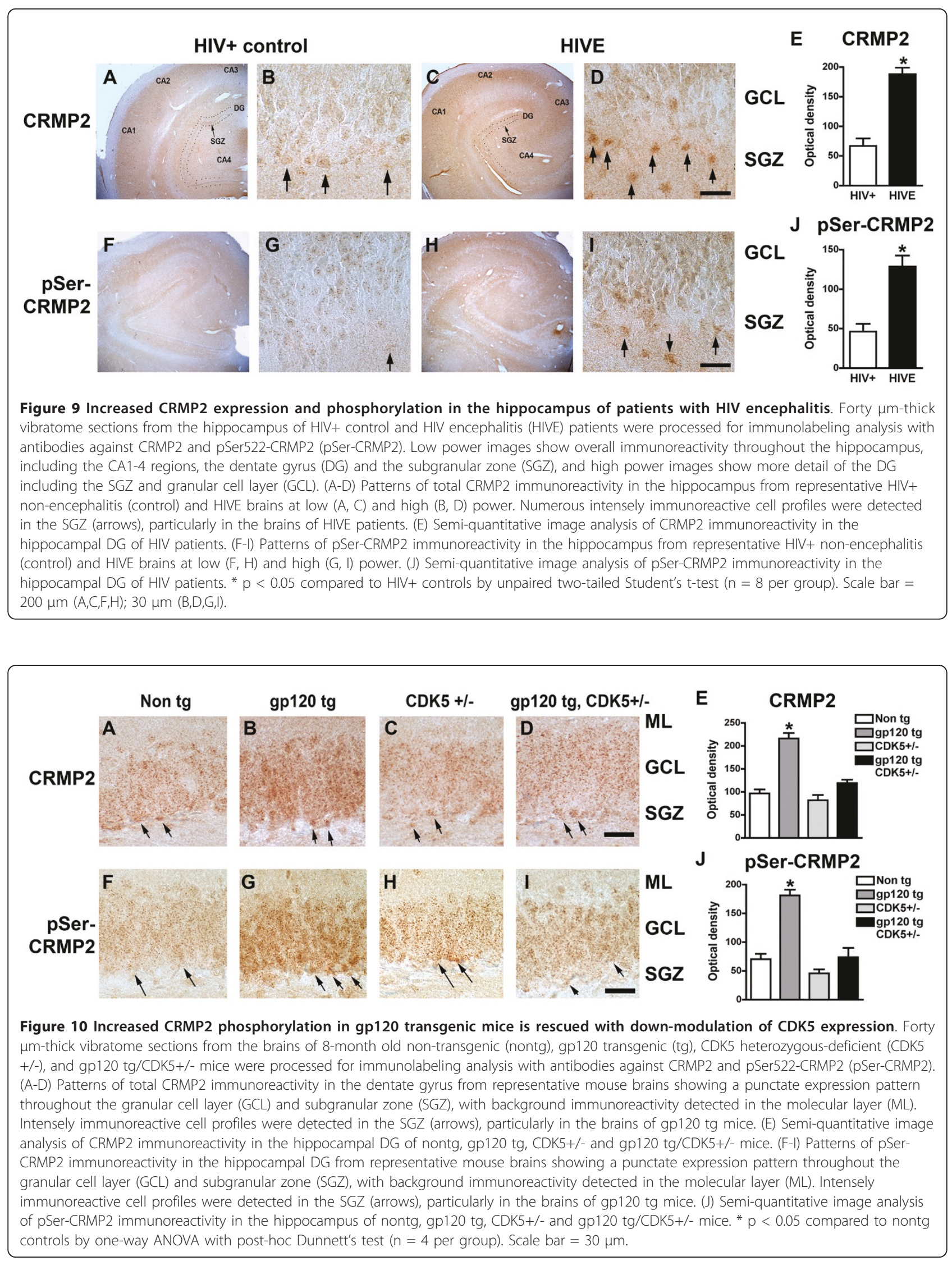
abnormal CDK5 activation in an in vitro model of adult neurogenesis contributes to defective neurite outgrowth during neuronal maturation. Down-regulation of CDK5 activity with the pharmacological inhibitor Roscovitine or siRNA knockdown, or expression of a non-phosphorylatable CRMP2 mutant construct (S522A) rescued the neurite defects associated with abnormal activation of CDK5 in NPC-derived neural progeny.

In support of a role for CRMP2 in neurodegenerative pathways, previous studies have revealed that CRMP2 is hyperphosphorylated as a result of abnormal CDK5 activity in the brains of $\mathrm{AD}$ patients and in a transgenic mouse model of AD [20]. In AD brains, phosphorylated CRMP2 associates with damaged neurites and neurofibrillary tangles [21,22,34], and accumulates in neurons surrounding cortical amyloid plaques [35]. More recently, several proteomics-based studies in the brains of human HIV patients have identified differential expression of CRMP2 in cases with HIVE or HIV-associated dementia $[23,24]$. The present study reveals that levels of total and phosphorylated (Ser522) CRMP2 were significantly increased in neurogenic sites of the hippocampus from HIVE patients compared to non-encephalitic HIV+ controls, and similar results were observed in a mouse model of HIV-gp120 neurotoxicity. While the present study was focused on the effects of CDK5 activation and CRMP2 alterations in the neurogenic region of the hippocampus, it is of interest that we observed cells throughout the SGZ and the granular cell layer displaying increased CRMP2 immunoreactivity and phosphorylation, particularly in the gp120 tg mouse model. This suggests that mature neuronal cell populations in addition to NPCs may be vulnerable to CDK5-mediated abnormal CRMP2 phosphorylation. Our results in primary hippocampal neurons also support this possibility, however future studies in other mature neuronal cell types and alternative brain regions (e.g. cortex) will be necessary to elucidate whether CDK5-mediated alterations in CRMP2 is a common pathological feature of HIV-associated neurodegeneration.

In the tg mouse model, hippocampal CRMP2 hyperphosphorylation was reversed with genetic down-modulation of CDK5. Since CDK5 activity and phosphorylation of other substrates in mature neurons is increased in models of HIV neurotoxicity [8], under these conditions, heterozygous CDK5 deficiency may be protective by normalizing the activity of CDK 5 via moderate reduction of CDK5 expression levels. In support of this possibility, we have previously demonstrated that heterozygous CDK5 deficiency reverses hyperphosphorylation of tau in mature neurons in the frontal cortex of gp120 tg mice [8], and that CDK5 knock-down rescues neurogenic deficits in an animal model of AD [17]. The present study suggests that hyperphosphorylation of
CRMP2 may be an important feature of HIV-associated neurodegeneration, and identifies a potential link between abnormal activation of CDK5 and impaired neurogenesis that has been observed in animal models of HIV neurotoxicity [36], however future studies will be necessary to elucidate whether CRMP2 hyperphosphorylation plays a direct causative role in neurogenic or neurodegenerative alterations in the pathogenesis of HAND.

Given that CRMP2 is a known substrate of CDK5, along with the recent evidence that CDK5 and CRMP2 are dysregulated in HIVE $[8,23,24]$, activation of CDK5 and subsequent hyperphosphorylation of CRMP2 may be an important mediator of neurodegeneration in HIV infection. The results of the present study also reveal a new potential role for CRMP2 alterations in the mechanisms of defective adult neurogenesis in neurodegenerative disorders such as HIVE. This is in line with previous studies that have shown defective neurogenesis in HIV patients and in animal models of HIV neurotoxicity (gp120 tg and others) [13,36,37].

Although the physiological function of CRMP2 has been associated with neuronal development and neurite outgrowth [38,39], the effects of abnormal CDK5-directed CRMP2 hyperphosphorylation in adult neurogenesis have not been previously investigated. CRMP2 (also known as Dpysl2) is a signaling protein that has been shown to play a role in growth cone collapse and axon development [40]. It is a member of a group of related proteins including CRMP1 and CRMP4, all of which are highly abundant in the brain. CRMP2 has no known enzymatic activity [41], however its C-terminal region is highly Ser and Thr-rich, and is targeted for phosphorylation by a number of kinases, including CDK5, GSK3 $\beta$ $[28,42]$, Rho kinase [43,44], and Fyn kinase [26], among others. Phosphorylation of CRMP2 has been reported for at least five amino acid residues in the C-terminal region, namely Thr509, Thr514, Ser518, Ser522 and Thr555. Phosphorylation by CDK5 at Ser522 acts as a priming site for subsequent phosphorylation by GSK3 $\beta$ at Thr514 [25,28], while other residues can be phosphorylated independently of CDK5 activity. It is possible that manipulation of phosphorylation at residues other than Ser522 of CRMP2 might also regulate neurite outgrowth, however because CDK5 is the only kinase that has been reported to phosphorylate the Ser522 residue, and since phosphorylation at this site acts a gatekeeper that regulates subsequent phosphorylation at other epitopes, we focused our studies on evaluating the specific role of phosphorylation at the Ser522 residue.

In support of a pivotal role for CDK5 in the phosphorylation of CRMP2, a previous study demonstrated that CRMP2 was not phosphorylated in $c d k 5-/-$ embryonic brain using an antibody that recognizes several 
phosphorylated epitopes of CRMP2 (Thr509, Ser518, and Ser522) [27]. Furthermore, the association of CRMP2 with tubulin was enhanced in $c d k 5$-/- brains compared to wild-type brain, supporting the role of CDK5-mediated phosphorylation of CRMP2 in modulating CRMP2-tubulin interactions [27]. The functional effects of phosphorylation at the other Ser/Thr residues of CRMP2 is not entirely clear, however previous studies have shown that some of these post-translational modifications dramatically modulate protein-protein interactions between CRMP2 and its binding partners, including tubulin heterodimers [30]. Of interest, previous studies have also shown that CDK5 and p35 can associate with microtubules $[45,46]$, and CDK5 substrates include a number of microtubule-associated proteins.

The results of the present study show that abnormal activation of CDK5 and subsequent hyperphosphorylation of CRMP2 may have detrimental effects on structural elements of developing neurons, such as microtubules and cytoskeletal organization. In support of this possibility, we showed that the alterations in neurite outgrowth and CRMP2 phosphorylation in p35overexpressing NPC-derived neural progeny were accompanied by disrupted microtubule organization compared to controls. Live-cell imaging of polymerized tubulin, and ultrastructural analysis of microtubule structure in NPC-derived neural progeny revealed diffuse, mesh-like microtubule distribution under conditions of p35-mediated abnormal CDK5 activation. This was in stark contrast to more robust microtubules that were observed under normal differentiation conditions. Furthermore, control NPC-derived neural progeny displayed rapid re-polymerization of microtubules following chemical disruption with the microtubule-destabilization agent, nocodazole, while cultures expressing p35 showed only minimal re-polymerization even after further incubation post-washout. These observations are in line with previous studies suggesting that the mechanisms downstream of abnormal CDK5 activation may involve the destabilization of microtubules [47] and that CRMP2 plays a role in regulating microtubule dynamics [48]. Together, this supports a role for CDK5-mediated CRMP2 hyperphosphorylation in the mechanisms of defective adult neurogenesis in neurodegenerative conditions such as HIVE.

\section{Conclusions}

Here we have shown that CRMP2 is a molecular mediator of abnormal CDK5 activation and impaired neuronal maturation in an in vitro model of adult neurogenesis. Abnormal activation of CDK5 by p35 overexpression, or under conditions of HIV protein-mediated toxicity, results in hyperphosphorylation of CRMP2 in an in vitro model of adult neurogenesis. The relevance of these results to disease mechanisms is supported by the observations of upregulated CRMP2 levels and phosphorylation in the brains of patients with HIVE and an in vivo model of HIV-gp120 mediated neurotoxicity. The microtubule abnormalities that accompanied CDK5 activation and CRMP2 hyperphosphorylation in the in vitro model support the possibility that alterations in p35/ CDK5/CRMP2 signaling contribute to impaired neurogenesis by disturbing microtubule integrity in maturing neurons. Targeting the CDK5/CRMP2 pathway for therapeutic intervention might provide new approaches to ameliorate the neurogenic alterations associated with neurodegenerative conditions including HIVE.

\section{Methods}

Neuronal progenitor cell and primary neuronal cell culture and in vitro modeling of abnormal CDK5 activation in progenitor cell and mature neuron populations

Adult rat hippocampal (ARH) NPCs (Millipore, Temecula, CA) were cultured routinely for expansion essentially as previously described [49] with some modifications. Briefly, cells were grown for expansion in DMEM/F12 media (Mediatech, Manassas, VA) containing B27 supplement, $1 \mathrm{X}$ L-glutamine and $1 \mathrm{X}$ antibioticantimycotic (all from Invitrogen, Carlsbad, CA). For induction of neuronal differentiation, cells were plated onto poly-ornithine/laminin (Sigma-Aldrich, St. Louis, MO) coated plates or coverslips and transferred the next day to differentiation media containing N2 supplement (Invitrogen), $1 \mu \mathrm{M}$ all-trans retinoic acid (SigmaAldrich), $5 \mu \mathrm{M}$ forskolin (Sigma-Aldrich) and 1\% FBS. Cells were differentiated for four days, and fresh differentiation media was added at day 2 . It should be noted that this differentiation procedure generates heterogeneous cultures, and therefore we refer to the cells derived from the differentiation process as "NPC-derived neural progeny."

To induce CDK5/p35 activity in vitro, cells were infected on day 2 of differentiation with adenovirus expressing human p35 or GFP control (Vector Biolabs, Philadelphia, PA) at a multiplicity of infection (MOI) of 30. Additional control experiments were performed with cells transfected using BPfectin according to the manufacturer's guidelines (Biopioneer, San Diego, CA) with a plasmid expressing myc-tagged p35 (Addgene plasmid 1347, deposited by Dr. Li-Huei Tsai, Picower Institute, Cambridge, MA). Two days after infection or transfection with p35, cells were processed for immunoblot analysis with total cell lysates or immunocytochemical analysis with fixed cells on coverslips.

Similar experiments were performed in rat hippocampal primary neurons (ScienCell Research Laboratories, 
Carlsbad, CA) cultured on poly-lysine-coated plates and glass coverslips according to the supplier's recommendations in Neuronal Medium (ScienCell). For induction of CDK5/p35 activity, cells were infected two days after plating with p35-adv at an MOI of 100. A higher MOI was selected for the primary neuronal experiments because these cells were plated at a lower density than NPCs in culture. Two days after infection, cells were prepared for immunoblot or immunocytochemical analyses. A subset of experiments were performed with NPCs and primary hippocampal neurons where uninfected cells or cells infected p35-adv were exposed to recombinant gp120 protein for 24-48 hrs (100-200 ng/ mL, LAV IIIB, Protein Sciences Corp., Meriden, CT) to model HIV protein associated neurotoxicity in vitro. At the conclusion of the in vitro experiments, cells were lysed for immunoblot analysis, or cells on coverslips were fixed in $4 \%$ paraformaldehyde (PFA) in phosphatebuffered saline (PBS) for immunocytochemical analysis.

\section{Site-directed mutagenesis and generation of hCRMP2 mutant constructs}

The wild-type hCRMP2/pCMV6-XL4 plasmid DNA (Origene, Rockville, MD) was maintained in E. coli TOP10 cells $\left(\mathrm{dam}^{+} / \mathrm{dcm}^{+}\right.$strain). Using plasmid DNA isolated from the $E$. coli strain as a template, site-directed mutagenesis of $h C R M P 2$ was carried out using a QuickChange $^{\circledR}$ Lightning Site-Directed Mutagenesis Kit (Stratagene, La Jolla, CA) with the primers 5'-ctcggccaagacggctcctgccaagcag-3' (sense) and 5'-ctgcttggcaggagccgtcttggccgag-3' (antisense) for S522A single point mutation. The PCR reaction was performed according to the manufacturer's instructions, with 10-min extension cycles at $68^{\circ} \mathrm{C}$. In order to remove the parental DNA template, PCR products were subjected to DpnI restriction enzyme digestion reaction and then directly transformed into E. coli TOP10 cells. After selection on LB medium supplemented with ampicillin, plasmid DNA was extracted from positive transformants using a QIAprep Spin Miniprep kit (Qiagen) according to the manufacturer's instructions. Purified plasmids were subjected to DNA sequencing with hCRMP2 specific primers: 5'-atcaaggcaaggagcaggct-3' (sense), and 5'aatgttgtcatcaatctgagcacca-3' (antisense). After sequence confirmation, the pCMV6-XL4 vector constructs containing either the wild-type hCRMP2, or the S522A construct were used for transformation and amplification in XL10 E. coli cells (Stratagene).

Cell culture treatments-Pharmacological treatments, and siRNA and CRMP2 plasmid DNA transfection

For inhibition of CDK5 activity, cells were treated at day 2 of differentiation with either the pharmacological CDK5 inhibitor Roscovitine (1-10 $\mu \mathrm{M}$, Calbiochem, San
Diego, CA) or transfected with siRNA against CDK5, CRMP2, or control non-targeting fluorescent-tagged siRNA (37.5-150 ng, $5 \mathrm{nM}$ final concentration, Qiagen, Valencia, CA) using the HiPerfect transfection reagent (Qiagen) according to the manufacturer's protocol. For each target, at least two different siRNAs were tested, and the one with the highest efficacy was selected for subsequent experiments.

For overexpression of wild-type or mutant human (h) CRMP2 in NPCs, cells were differentiated from day 0 in medium without antibiotics, and transfected on day 2 of differentiation (6 hrs prior to virus infection) with pCMV6-XL4 plasmids hCRMP2-WT, or hCRMP2S522A, or pCMV-GFP control. Transfection was performed using Lipofectamine 2000 transfection reagent (Invitrogen) at a concentration of $2.5 \mu \mathrm{L} / \mathrm{mL}$ according to the manufacturer's instructions. For transfection of cells in 6-well plates, $4.0 \mu \mathrm{g}$ plasmid DNA was applied per well, and for transfection of cells on coverslips in 6 $\mathrm{cm}$ dishes, $8.0 \mu \mathrm{g}$ plasmid DNA was applied per dish. Six hrs after transfection, fresh differentiation medium without antibiotics was applied with or without viral vectors. Cells were then lysed for biochemical analyses or fixed for immunocytochemical analysis. For disruption of microtubules, cells were treated with nocodazole ( $5 \mu \mathrm{g} / \mathrm{mL}$, Sigma-Aldrich) for $3 \mathrm{hrs}$. Then, cultures were washed with differentiation media three times, followed by incubation in fresh media for $10 \mathrm{mins}, 20 \mathrm{mins}$ or 30 mins. Cells were then fixed with glutaraldehyde for tubulin immunofluorescence and neurite outgrowth analysis.

\section{Neurite outgrowth studies}

A subset of cultured cells on coverslips were fixed in a solution containing glutaraldehyde, which better preserves the cytoskeleton for optimal visualization of neurites using immunofluorescence with an antibody against $\beta$-Tubulin. For this purpose, a fixation procedure was used essentially as previously described by Desai and Mitchison [50]. Briefly, media was gently aspirated from cells growing on glass coverslips, and then cells were extracted for $30 \mathrm{sec}$ in cytoskeletal buffer (CB, $80 \mathrm{mM}$ PIPES pH 6.8, $1 \mathrm{mM} \mathrm{MgCl}_{2}$, 4 mM EGTA) containing $0.5 \%$ freshly added Triton-X 100. Glutaraldehyde (Electron Microscopy Sciences, Hatfield, PA) was immediately added to the $\mathrm{CB}$ on the coverslips at a final concentration of $0.5 \%$. Coverslips were incubated for 10 $\min$ at $37^{\circ} \mathrm{C}$. Fixative was then removed and a freshlyprepared solution of $0.1 \% \mathrm{NaBH}_{4}$ in PBS was added and samples were incubated for $7 \mathrm{~min}$ at room temperature to quench free glutaraldehyde. Coverslips were washed at least 3 times in PBS to remove the $\mathrm{NaBH}_{4}$, and samples were then processed for $\beta$-Tubulin immunofluorescence analysis. 
For $\beta$-Tubulin immunofluorescence and neurite outgrowth studies, NPC-derived neural progeny growing on coverslips were fixed with glutaraldehyde and incubated with a mouse monoclonal primary anti- $\beta$-Tubulin antibody (1:250, Clone B2.1, Sigma-Aldrich) for $1 \mathrm{hr}$ at room temperature and detected with FITC-conjugated secondary antibodies (1:75, Vector Laboratories). $\beta$ Tubulin-labeled coverslips were mounted under glass coverslips with ProLong Gold antifade reagent with DAPI (Invitrogen) and imaged with a fluorescent digital microscopy (Olympus). For analysis of neurite outgrowth of NPC-derived neural progeny immunolabeled with $\beta$-Tubulin, neurites were traced and lengths were measured using the Image J Program (NIH, Bethesda, MD) with the NeuronJ Plugin [51].

\section{Live-cell staining and imaging of polymerized tubulin in NPC-derived neural progeny}

For staining of polymerized tubulin in live cells, NPCderived neural progeny treated with vehicle control or p35-adv were grown on glass coverslips in 12-well plates as described above, and incubated with Tubulin Tracker Green (Invitrogen) according to the manufacturer's guidelines. Briefly, at day 4 of differentiation, media was removed from the cultures, and replaced with $1 \mathrm{~mL}$ of warm HBSS (containing $\mathrm{Ca}, \mathrm{Mg}$, and $4 \mathrm{mM}$ HEPES buffer [HBSS/HEPES buffer]) with $150 \mathrm{nM}$ Tubulin Tracker reagent. Plates were incubated for an additional $10 \mathrm{mins}$ at $37^{\circ} \mathrm{C}$, and then washed three times in warm HBSS/ HEPES buffer. Coverslips were rapidly transferred to slides and imaged within 5 mins on a digital fluorescent Olympus microscope.

\section{Electron microscopy analysis}

Briefly, as previously described [52], NPCs were plated in $35 \mathrm{~mm}$ dishes with a coverslip in the bottom (MatTek, Ashland, MA) and infected with p35-adv as described in the cell culture conditions. After 4 days of differentiation, cells were fixed in $1 \%$ glutaraldehyde in media, then fixed in osmium tetraoxide and embedded in epon araldite. Once the resin hardened, blocks with the cells were detached from the coverslips and mounted for sectioning with an ultramicrotome (Leica, Germany). Grids were analyzed with a Zeiss OM 10 electron microscope as previously described [53].

\section{HIV cases and neuropathological assessment}

For the present study HIV+ cases with and without encephalitis were selected from a cohort of $43 \mathrm{HIV}+$ subjects from the HIV Neurobehavioral Research Center (HNRC) and California NeuroAIDS Tissue Network (CNTN) at the University of California, San Diego. Subjects were excluded in these analyses if they had a history of CNS opportunistic infections or non-HIV-related developmental, neurologic, psychiatric, or metabolic conditions that might affect CNS functioning (e.g., loss of consciousness exceeding 30 minutes, psychosis, substance dependence). A total of 16 age-matched cases were identified with and without encephalitis $(n=8$ per group), and without other complications, for inclusion in the present study (Table 2). All cases had neuromedical and neuropsychological examinations within a median of 12 months before death. Most patients died as a result of acute bronchopneumonia or septicemia, and autopsy was performed within $24 \mathrm{hrs}$ of death (Table 2). Autopsy findings were consistent with AIDS, and the associated pathology was most frequently due to systemic CMV, Kaposi sarcoma, and liver disease. In all cases, neuropathological assessment was performed in paraffin sections from the frontal, parietal, and temporal cortices, and the hippocampus, basal ganglia and brainstem stained with $\mathrm{H} \& \mathrm{E}$, or immunolabeled with antibodies against p24 and glial fibrillary acidic protein (GFAP, marker of astrogliosis) [54,55]. The diagnosis of HIVE was based on the presence of microglial nodules, astrogliosis, HIV-p24 positive cells, and myelin pallor. Formalin-fixed sections and frozen brain samples were obtained from the hippocampus of HIV and HIVE cases for biochemical analysis. Brain tissue from HIV-infected subjects without evidence of neuroinflammation provides a close control for the systemic effects of HIV infection in the absence of neurodegenerative changes, and previous studies have shown that the CDK5 pathway is dysregulated specifically in cases with encephalitis compared to HIV-infected non-encephalitis cases $[8,56]$. For these reasons, and due to the scarcity of tissue samples available from age-matched non-HIV infected control subjects, HIV-positive cases without neuroinflammatory changes were used for comparison with cases with HIVE.

\section{Generation of GFAP-gp120 tg mice and crosses with CDK5-deficient mice}

For studies of CDK5 activation in an animal model of HIV-protein mediated neurotoxicity, tg mice expressing high levels of gp120 under the control of the GFAP promoter were used [57]. These mice develop neurodegeneration accompanied by astrogliosis, microgliosis [57], and memory deficits in the water maze [58]. To study the effects of genetic CDK5 inhibition on CRMP2 expression and phosphorylation in vivo, CDK5 heterozygous-deficient mice $\left(\mathrm{CDK} 5^{+/-}\right.$) [59] were crossed with the GFAP-gp120 tg mice as previously described [8]. Full ablation of both copies of CDK5 $\left(\mathrm{CDK} 5^{-/-}\right)$causes severe neurodevelopmental alterations, so in order to study CDK5 knockdown in the adult mouse brain, the $\mathrm{CDK} 5^{+/-}$animals were used as a model of reduced CDK5 activity. For in vivo studies, brain sections from 
8-month old nontg, CDK $5^{+/-}$, gp120 tg, or gp120 tg/ $\mathrm{CDK} 5^{+/-}$crossed mice ( $\mathrm{n}=4$ mice per group) were used for biochemical analysis of CRMP2 expression and phosphorylation.

\section{Tissue processing}

In accordance with NIH guidelines for the humane treatment of animals, mice were anesthetized with chloral hydrate and flush-perfused transcardially with $0.9 \%$ saline. Brains were removed and divided sagittally. One hemibrain was post-fixed in phosphate-buffered $4 \%$ paraformaldehyde at $4^{\circ} \mathrm{C}$ for $48 \mathrm{hrs}$ and sectioned at 40 $\mu \mathrm{m}$ with a Vibratome 2000, while the other hemibrain was snap frozen and stored at $-70^{\circ} \mathrm{C}$ for protein analysis. All experiments described were approved by the animal subjects committee at the University of California at San Diego (UCSD) and were performed according to NIH recommendations for animal use.

\section{Immunoblot analysis}

For immunoblot analysis, adherent cells in culture or mouse or human brain samples $(0.1 \mathrm{~g})$ were lysed in buffer composed of $10 \mathrm{mM}$ Tris- $\mathrm{HCl}$ (pH 7.4), $150 \mathrm{mM}$ $\mathrm{NaCl}, 5 \mathrm{mM}$ EDTA (TNE) containing 1\% Triton-X 100, homogenized using a microgrinder, and centrifuged at $10,000 \mathrm{rpm}$ for 10 minutes to clear insoluble material. The supernatant was harvested, and protein content in the total cell lysates was determined using the bicinchoninic acid protein assay kit (Pierce, Rockford, IL). Then, $20 \mu \mathrm{g}$ of each sample was separated by gel electrophoresis on 4-12\% Bis-Tris gels (Invitrogen) and blotted onto $0.45 \mu \mathrm{m}$ PVDF membranes (Millipore, Temecula, CA). All immunoblots were incubated in primary antibodies diluted in 5\% BSA in PBS-Tween (PBS-T) overnight at $4{ }^{\circ} \mathrm{C}$. Immunoblots were probed with rabbit polyclonal antibodies against phosphorylated (pSer522) CRMP2 (1:1000, ECM Biosciences, Versailles, KY), phosphorylated (pThr514) CRMP2 (1:1000, Cell Signaling Technology, Danvers, MA), phosphorylated (pThr555) CRMP2 (1:1000, ECM Biosciences), total CRMP2 (1:1000, Millipore), p35/p25 (1:500, C-19, SantaCruz Biotechnology, Santa Cruz, CA), CDK5 (1:500, C-8, SantaCruz Biotechnology), or GFP (1:1000, Millipore); or mouse monoclonal antibodies against $\beta$-III Tubulin (1:5000, clone Tuj1, Covance), $\beta$-Tubulin (1:1000, clone B2.1, Sigma-Aldrich), or GFAP (1:1000, Millipore).

For immunoblot analysis with a panel of additional phosphorylated or total CRMP proteins, blots were probed with antibodies described in Table 1 at a dilution of 1:1000 for all antibodies. All immunoblots were stripped and probed with an antibody against actin ( $\mathrm{C} 4$ clone, Millipore) as a loading control as previously described [53]. After incubation with primary antibodies, blots were incubated for 45 mins at room temperature in secondary antibodies diluted in 5\% non-fat milk with $1 \%$ BSA in PBS-T. Blots were developed with enhanced chemiluminescence (Perkin-Elmer, Waltham, MA), and images were obtained and semi-quantitative analysis was performed using the VersaDoc gel imaging system and Quantity One software (Bio-Rad, Hercules, CA).

\section{Immunocytochemistry and image analysis}

For immunocytochemical analysis, briefly, as previously described [60], cells on coverslips were fixed in 4\% paraformaldehyde (PFA) in PBS and washed with Tris buffered saline (TBS, pH 7.4). For single-label immunostaining, coverslips were pre-treated in $3 \%$ $\mathrm{H}_{2} \mathrm{O}_{2}$, blocked with $10 \%$ serum (Vector Laboratories, Burlingame, CA), and incubated with a rabbit polyclonal primary antibody against phospho-CRMP2 (Ser522, 1:1500, ECM Biosciences) diluted in PBS-T, and detected with the Tyramide Signal Amplification ${ }^{\mathrm{TM}}$ Direct (Red) system (NEN Life Sciences, Boston, MA). Immunolabeled coverslips were mounted under glass coverslips with ProLong Gold antifade reagent with DAPI (Invitrogen) and imaged with a Zeiss 63X (N.A. 1.4) objective on an Axiovert 35 microscope (Zeiss, Germany) with an attached MRC1024 laser scanning confocal microscope system (BioRad) [61]. All samples were processed simultaneously under the same conditions and the experiments were performed twice to assess reproducibility. To confirm the specificity of primary antibodies, control experiments were performed where sections were incubated overnight in the absence of primary antibody (deleted) or primary antibody pre-incubated with blocking peptide.

For immunocytochemical analysis in human or mouse brain tissue, briefly as previously described [61] vibratome sections from the hippocampus ( $40 \mu \mathrm{m}$ thick) of the HIV patients or from nontg, gp120 tg or CDK $5^{+/-}$ mice were incubated with antibodies against phosphoCRMP2 (Ser522, 1:300, ECM Biosciences) or CRMP2 (1:300, Millipore). Primary antibody incubation was followed by incubation with secondary biotinylated IgG, then avidin-HRP and diaminobenzidine (DAB) detection as previously described [8]. Immunostained sections were imaged with a digital Olympus microscope and assessment of levels of immunoreactivity was performed utilizing the Image-Pro Plus program (Media Cybernetics, Silver Spring, MD).

For double-labeling analysis, coverslips or brain sections were incubated with a rabbit polyclonal primary antibody against phospho-CRMP2 (Ser522, 1:1500, ECM Biosciences) or CRMP2 (1:1500, Millipore) detected with Tyramide Red. The next day samples were colabeled with mouse monoclonal antibodies against $\beta$-III Tubulin (1:250, Tuj1 clone, Covance) or MAP2 (1:100, 
Millipore), or a goat polyclonal antibody against the immature neuronal marker DCX (1:100, Santa Cruz Biotechnology), detected with FITC-conjugated secondary antibodies (1:75, Vector Laboratories). Samples were mounted and imaged as described above for singlelabeling immunofluorescence analysis. For each sample a total of three sections (10 digital images per section at $400 \times$ ) were analyzed in order to estimate the average number of immunolabeled cells per unit area $\left(\mathrm{mm}^{2}\right)$ and the average intensity of the immunostaining (corrected optical density).

\section{Statistical Analysis}

All experiments were performed blind coded and in triplicate. Values in the figures are expressed as means \pm SEM. To determine the statistical significance, values were compared by unpaired two-tailed Student's t-test or by one-way ANOVA with post-hoc Dunnett's test when comparing differences to controls, or by one-way ANOVA with post-hoc Tukey-Kramer test when comparisons were made among groups. The differences were considered to be significant if $\mathrm{p}$ values were less than 0.05 .

\section{Additional material}

Additional file 1: Supplementary Figures 1-3 and legends. Three supplementary figures and the corresponding figure legends are provided as additional materials with the main manuscript submission.

\section{List of abbreviations}

AD: Alzheimer's disease; CDK5: cyclin-dependent kinase-5; HIVE: HIV encephalitis; NPCs: neural progenitor cells; SVZ: subventricular zone; DG: dentate gyrus; CRMP2: collapsin-response mediator protein-2; tg: transgenic; DCX: doublecortin; SGZ: subgranular zone; nontg: non-transgenic; PFA: paraformaldehyde; PBS: phosphate-buffered saline; HAND: HIV associated neurocognitive disorders

\section{Acknowledgements}

This work was supported by NIH Grants AG022074, AG018440, NS057096, AG010435, AG005131, AG011385, MH062962, MH076681, MH045294, MH059745, MH058164, DA026306, and California NeuroAIDS Tissue Network U01 MH083506. The HIV Neurobehavioral Research Center (HNRC) is supported by Center award MH062512 from NIMH. The authors also wish to thank Dr. Paula Desplats for critical discussion and valuable suggestions during the manuscript revision process.

\section{Author details}

'Department of Neurosciences; University of California, San Diego; 9500 Gilman Drive, La Jolla, CA 92093-0624, USA. ²Department of Pathology; University of California, San Diego; 9500 Gilman Drive, La Jolla, CA 92093 USA. ${ }^{3}$ Department of Psychiatry; University of California, San Diego; 9500 Gilman Drive, La Jolla, CA 92093, USA.

\section{Authors' contributions}

LC participated in the conception, design and coordination of the study, carried out the in vitro experiments and biochemical assays, and drafted the manuscript. RR participated in the immunoblot studies, carried out the immunohistochemical studies in human and mouse brain sections, and provided critical review of the manuscript. CP carried out the immunocytochemical assays for in vitro experiments. WD provided human brain tissue samples and prepared tissue samples for immunoblot analysis. MTM carried out the electron microscopy studies. CA participated in the design of the study and provided critical review of the manuscript. ER carried out the breeding and crosses of transgenic mouse lines. EM conceived of the study, and participated in its design and coordination and helped to draft the manuscript. All authors read and approved the final manuscript.

\section{Competing interests}

The authors declare that they have no competing interests.

Received: 15 April 2011 Accepted: 24 September 2011

Published: 24 September 2011

\section{References}

1. Lee HG, Moreira PI, Zhu X, Smith MA, Perry G: Staying connected: synapses in Alzheimer disease. Am J Pathol 2004, 165:1461-1464.

2. Masliah E, Heaton RK, Marcotte TD, Ellis RJ, Wiley CA, Mallory M, Achim CL, McCutchan JA, Nelson JA, Atkinson JH, Grant I: Dendritic injury is a pathological substrate for human immunodeficiency virus-related cognitive disorders. HNRC Group. The HIV Neurobehavioral Research Center. Ann Neurol 1997, 42:963-972.

3. Kaul M, Garden GA, Lipton SA: Pathways to neuronal injury and apoptosis in HIV-associated dementia. Nature 2001, 410:988-994.

4. Liu F, Su Y, Li B, Zhou Y, Ryder J, Gonzalez-DeWhitt P, May PC, Ni B: Regulation of amyloid precursor protein (APP) phosphorylation and processing by p35/Cdk5 and p25/Cdk5. FEBS Lett 2003, 547:193-196.

5. Patrick GN, Zukerberg L, Nikolic M, de la Monte S, Dikkes P, Tsai LH: Conversion of p35 to p25 deregulates Cdk5 activity and promotes neurodegeneration. Nature 1999, 402:615-622.

6. Wang GR, Shi S, Gao C, Zhang BY, Tian C, Dong CF, Zhou RM, Li XL, Chen C, Han J, Dong XP: Changes of tau profiles in brains of the hamsters infected with scrapie strains $263 \mathrm{~K}$ or 139 A possibly associated with the alteration of phosphate kinases. BMC Infect Dis 2010, 10:86.

7. Lee KY, Clark AW, Rosales JL, Chapman K, Fung T, Johnston RN: Elevated neuronal Cdc2-like kinase activity in the Alzheimer disease brain. Neurosci Res 1999, 34:21-29.

8. Patrick C, Crews L, Desplats P, Dumaop W, Rockenstein E, Achim CL, Everall IP, Masliah E: Increased CDK5 Expression in HIV Encephalitis Contributes to Neurodegeneration via Tau Phosphorylation and Is Reversed with Roscovitine. Am J Pathol 2011, 178:1646-1661.

9. Jin K, Peel AL, Mao XO, Xie L, Cottrell BA, Henshall DC, Greenberg DA: Increased hippocampal neurogenesis in Alzheimer's disease. Proc Natl Acad Sci USA 2004, 101:343-347.

10. Boekhoorn K, Joels M, Lucassen PJ: Increased proliferation reflects glial and vascular-associated changes, but not neurogenesis in the presenile Alzheimer hippocampus. Neurobiol Dis 2006, 24:1-14.

11. Li B, Yamamori H, Tatebayashi Y, Shafit-Zagardo B, Tanimukai H, Chen S, lqbal K, Grundke-lqbal I: Failure of neuronal maturation in Alzheimer disease dentate gyrus. J Neuropathol Exp Neurol 2008, 67:78-84.

12. Crews L, Adame A, Patrick C, Delaney A, Pham E, Rockenstein E, Hansen L, Masliah E: Increased BMP6 levels in the brains of Alzheimer's disease patients and APP transgenic mice are accompanied by impaired neurogenesis. J Neurosci 2010, 30:12252-12262.

13. Okamoto S, Kang YJ, Brechtel CW, Siviglia E, Russo R, Clemente A, Harrop A, McKercher S, Kaul M, Lipton SA: HIV/gp120 Decreases Adult Neural Progenitor Cell Proliferation via Checkpoint Kinase-Mediated Cell-Cycle Withdrawal and G1 Arrest. Cell Stem Cell 2007, 1:230-236.

14. Kaul M: HIV's double strike at the brain: neuronal toxicity and compromised neurogenesis. Front Biosci 2008, 13:2484-2494.

15. Jessberger S, Aigner S, Clemenson GD Jr, Toni N, Lie DC, Karalay O, Overall R, Kempermann G, Gage FH: Cdk5 regulates accurate maturation of newborn granule cells in the adult hippocampus. PLOS Biol 2008, 6: e272.

16. Lagace DC, Benavides DR, Kansy JW, Mapelli M, Greengard P, Bibb JA, Eisch AJ: Cdk5 is essential for adult hippocampal neurogenesis. Proc Natl Acad Sci USA 2008, 105:18567-18571. 
17. Crews L, Patrick C, Adame A, Rockenstein E, Masliah E: Modulation of aberrant CDK5 signaling rescues impaired neurogenesis in models of Alzheimer's disease. Cell Death Dis 2011, 2:e120.

18. Sahlgren CM, Mikhailov A, Vaittinen S, Pallari HM, Kalimo H, Pant HC, Eriksson JE: Cdk5 regulates the organization of Nestin and its association with p35. Mol Cell Biol 2003, 23:5090-5106.

19. Matsubara M, Kusubata M, Ishiguro K, Uchida T, Titani K, Taniguchi H: Sitespecific phosphorylation of synapsin I by mitogen-activated protein kinase and Cdk5 and its effects on physiological functions. J Biol Chem 1996, 271:21108-21113

20. Soutar MP, Thornhill P, Cole AR, Sutherland C: Increased CRMP2 phosphorylation is observed in Alzheimer's disease; does this tell us anything about disease development? Curr Alzheimer Res 2009, 6:269-278.

21. Cole AR, Noble W, van Aalten L, Plattner F, Meimaridou R, Hogan D, Taylor M, LaFrancois J, Gunn-Moore F, Verkhratsky A, Oddo S, LaFerla F, Giese KP, Dineley KT, Duff K, Richardson JC, Yan SD, Hanger DP, Allan SM, Sutherland C: Collapsin response mediator protein-2 hyperphosphorylation is an early event in Alzheimer's disease progression. J Neurochem 2007, 103:1132-1144.

22. Yoshida $H$, Watanabe $A$, Ihara $Y$ : Collapsin response mediator protein-2 is associated with neurofibrillary tangles in Alzheimer's disease. J Biol Chem 1998, 273:9761-9768.

23. Gelman BB, Nguyen TP: Synaptic proteins linked to HIV-1 infection and immunoproteasome induction: proteomic analysis of human synaptosomes. J Neuroimmune Pharmacol 2010, 5:92-102.

24. Zhou L, Diefenbach E, Crossett B, Tran SL, Ng T, Rizos H, Rua R, Wang B, Kapur A, Gandhi K, Brew BJ, Saksena NK: First evidence of overlaps between HIV-Associated Dementia (HAD) and non-viral neurodegenerative diseases: proteomic analysis of the frontal cortex from HIV+ patients with and without dementia. Mol Neurodegener 2010, 5:27.

25. Cole AR, Causeret F, Yadirgi G, Hastie CJ, McLauchlan H, McManus EJ, Hernandez F, Eickholt BJ, Nikolic M, Sutherland C: Distinct priming kinases contribute to differential regulation of collapsin response mediator proteins by glycogen synthase kinase-3 in vivo. J Biol Chem 2006, 281:16591-16598.

26. Uchida Y, Ohshima T, Yamashita N, Ogawara M, Sasaki Y, Nakamura F, Goshima Y: Semaphorin3A signaling mediated by Fyn-dependent tyrosine phosphorylation of collapsin response mediator protein 2 at tyrosine 32. J Biol Chem 2009, 284:27393-27401.

27. Goshima Y, Sasaki Y, Uchida Y, Yamashita M, Nakamura F: CRMP family protein: Novel targets for CDK5 that regulate axon guidance, synapse maturation, and cell migration. In Cyclin dependent kinase 5 (Cdk5). Edited by: Ip NY, Tsai LH. New York: Springer; 2008:9-24.

28. Uchida $Y$, Ohshima T, Sasaki $Y$, Suzuki H, Yanai S, Yamashita N, Nakamura F, Takei K, Ihara Y, Mikoshiba K, Kolattukudy P, Honnorat J, Goshima Y: Semaphorin3A signalling is mediated via sequential Cdk5 and GSK3 $\beta$ phosphorylation of CRMP2: implication of common phosphorylating mechanism underlying axon guidance and Alzheimer's disease. Genes Cells 2005, 10:165-179.

29. Cole AR, Soutar MP, Rembutsu M, van Aalten L, Hastie CJ, McLauchlan H, Peggie M, Balastik M, Lu KP, Sutherland C: Relative resistance of Cdk5phosphorylated CRMP2 to dephosphorylation. J Biol Chem 2008, 283:18227-18237.

30. Fukata $Y$, Itoh TJ, Kimura T, Menager C, Nishimura T, Shiromizu T, Watanabe $\mathrm{H}$, Inagaki N, Iwamatsu A, Hotani H, Kaibuchi K: CRMP-2 binds to tubulin heterodimers to promote microtubule assembly. Nat Cell Biol 2002, 4:583-591.

31. Wang $Y$, White MG, Akay C, Chodroff RA, Robinson J, Lindl KA, Dichter MA, Qian Y, Mao Z, Kolson DL, Jordan-Sciutto KL: Activation of cyclindependent kinase 5 by calpains contributes to human immunodeficiency virus-induced neurotoxicity. J Neurochem 2007 103:439-455.

32. Cruz JC, Tsai LH: A Jekyll and Hyde kinase: roles for Cdk5 in brain development and disease. Curr Opin Neurobiol 2004, 14:390-394.

33. Han P, Dou F, Li F, Zhang X, Zhang YW, Zheng H, Lipton SA, Xu H, Liao FF: Suppression of cyclin-dependent kinase 5 activation by amyloid precursor protein: a novel excitoprotective mechanism involving modulation of tau phosphorylation. J Neurosci 2005, 25:11542-11552.
34. Gu Y, Hamajima N, Ihara Y: Neurofibrillary tangle-associated collapsin response mediator protein-2 (CRMP-2) is highly phosphorylated on Thr509, Ser-518, and Ser-522. Biochemistry 2000, 39:4267-4275.

35. Petratos S, Li QX, George AJ, Hou X, Kerr ML, Unabia SE, Hatzinisiriou I, Maksel D, Aguilar MI, Small DH: The $\beta$-amyloid protein of Alzheimer's disease increases neuronal CRMP-2 phosphorylation by a Rho-GTP mechanism. Brain 2008, 131:90-108

36. Lee MH, Wang $T$, Jang MH, Steiner J, Haughey $N$, Ming GL, Song $H$, Nath A, Venkatesan A: Rescue of adult hippocampal neurogenesis in a mouse model of HIV neurologic disease. Neurobiol Dis 2011, 41:678-687.

37. Venkatesan A, Nath A, Ming GL, Song H: Adult hippocampal neurogenesis: regulation by HIV and drugs of abuse. Cell Mol Life Sci 2007, 64:2120-2132.

38. Charrier E, Reibel S, Rogemond V, Aguera M, Thomasset N, Honnorat J: Collapsin response mediator proteins (CRMPs): involvement in nervous system development and adult neurodegenerative disorders. Mol Neurobiol 2003, 28:51-64.

39. Rogemond V, Auger C, Giraudon P, Becchi M, Auvergnon N, Belin MF, Honnorat J, Moradi-Ameli M: Processing and nuclear localization of CRMP2 during brain development induce neurite outgrowth inhibition. $J$ Biol Chem 2008, 283:14751-14761.

40. Goshima Y, Nakamura F, Strittmatter P, Strittmatter SM: Collapsin-induced growth cone collapse mediated by an intracellular protein related to UNC-33. Nature 1995, 376:509-514

41. Wang LH, Strittmatter SM: Brain CRMP forms heterotetramers similar to liver dihydropyrimidinase. J Neurochem 1997, 69:2261-2269.

42. Cole AR, Knebel A, Morrice NA, Robertson LA, Irving AJ, Connolly CN, Sutherland C: GSK-3 phosphorylation of the Alzheimer epitope within collapsin response mediator proteins regulates axon elongation in primary neurons. J Biol Chem 2004, 279:50176-50180.

43. Arimura N, Inagaki N, Chihara K, Menager C, Nakamura N, Amano M Iwamatsu A, Goshima Y, Kaibuchi K: Phosphorylation of collapsin response mediator protein-2 by Rho-kinase. Evidence for two separate signaling pathways for growth cone collapse. J Biol Chem 2000, 275:23973-23980.

44. Arimura N, Menager C, Fukata Y, Kaibuchi K: Role of CRMP-2 in neuronal polarity. J Neurobiol 2004, 58:34-47.

45. Sobue K, Agarwal-Mawal A, Li W, Sun W, Miura Y, Paudel HK: Interaction of neuronal Cdc2-like protein kinase with microtubule-associated protein tau. J Biol Chem 2000, 275:16673-16680.

46. Hou Z, Li Q, He L, Lim HY, Fu X, Cheung NS, Qi DX, Qi RZ: Microtubule association of the neuronal p35 activator of Cdk5. J Biol Chem 2007 282:18666-18670.

47. Li G, Faibushevich A, Turunen BJ, Yoon SO, Georg G, Michaelis ML, Dobrowsky RT: Stabilization of the cyclin-dependent kinase 5 activator, p35, by paclitaxel decreases $\beta$-amyloid toxicity in cortical neurons. J Neurochem 2003, 84:347-362

48. Gu Y, Ihara Y: Evidence that collapsin response mediator protein-2 is involved in the dynamics of microtubules. J Biol Chem 2000, 275:17917-17920.

49. Ray J, Gage FH: Differential properties of adult rat and mouse brainderived neural stem/progenitor cells. Mol Cell Neurosci 2006, 31:560-573.

50. Desai A, Mitchison T: Fluorescence Procedures for the Actin and Tubulin Cytoskeleton in Fixed Cells.[http://mitchison.med.harvard.edu/protocols/ gen1.html], Accessed 8/25/2011..

51. Meijering $E$, Jacob M, Sarria JC, Steiner P, Hirling H, Unser M: Design and validation of a tool for neurite tracing and analysis in fluorescence microscopy images. Cytometry A 2004, 58:167-176.

52. Marongiu R, Spencer $B$, Crews L, Adame A, Patrick C, Trejo M, Dallapiccola B, Valente EM, Masliah E: Mutant Pink1 induces mitochondrial dysfunction in a neuronal cell model of Parkinson's disease by disturbing calcium flux. J Neurochem 2009, 108:1561-1574.

53. Rockenstein E, Mallory M, Mante M, Sisk A, Masliah E: Early formation of mature amyloid-b proteins deposits in a mutant APP transgenic model depends on levels of Ab1-42. J neurosci Res 2001, 66:573-582.

54. Achim $C L$, Heyes MP, Wiley CA: Quantitation of human immunodeficiency virus, immune activation factors, and quinolinic acid in AIDS brains. $J$ Clin Invest 1993, 91:2769-2775.

55. Masliah E, Achim C, Ge N, DeTeresa R, Terry R, Wiley C: Spectrum of human immunodeficiency virus-associated neocortical damage. AnnNeurol 1992, 32:321-329. 
56. Masliah E, Roberts ES, Langford D, Everall I, Crews L, Adame A, Rockenstein E, Fox HS: Patterns of gene dysregulation in the frontal cortex of patients with HIV encephalitis [Erratum appeared in J Neuroimmunol 2005, 162:197]. J Neuroimmunol 2004, 157:163-175.

57. Toggas S, Masliah E, Rockenstein E, Mucke L: Central nervous system damage produced by expression of the HIV-1 coat protein gp120 in transgenic mice. Nature 1994, 367:188-193.

58. D'Hooge R, Franck F, Mucke L, De Deyn PP: Age-related behavioural deficits in transgenic mice expressing the HIV-1 coat protein gp120. Eur J Neurosci 1999, 11:4398-4402.

59. Ohshima T, Ward JM, Huh CG, Longenecker G, Veeranna, Pant HC, Brady RO, Martin LJ, Kulkarni AB: Targeted disruption of the cyclindependent kinase 5 gene results in abnormal corticogenesis, neuronal pathology and perinatal death. Proc Natl Acad Sci USA 1996, 93:11173-11178.

60. Masliah E, Alford M, Adame A, Rockenstein E, Galasko D, Salmon D, Hansen LA, Thal LJ: A $1-42$ promotes cholinergic sprouting in patients with AD and Lewy body variant of AD. Neurology 2003, 61:206-211.

61. Masliah E, Rockenstein E, Veinbergs I, Mallory M, Hashimoto M, Takeda A, Sagara, Sisk A, Mucke L: Dopaminergic loss and inclusion body formation in a-synuclein mice: Implications for neurodegenerative disorders. Science 2000, 287:1265-1269.

doi:10.1186/1750-1326-6-67

Cite this article as: Crews et al:: Phosphorylation of collapsin response mediator protein-2 disrupts neuronal maturation in a model of adult neurogenesis: Implications for neurodegenerative disorders. Molecular Neurodegeneration 2011 6:67.

\section{Submit your next manuscript to BioMed Central and take full advantage of:}

- Convenient online submission

- Thorough peer review

- No space constraints or color figure charges

- Immediate publication on acceptance

- Inclusion in PubMed, CAS, Scopus and Google Scholar

- Research which is freely available for redistribution

Submit your manuscript at www.biomedcentral.com/submit 